\title{
The Deuterium Chemistry of the Early Universe
}

\section{Citation}

Stancil, P. C., S. Lepp, and A. Dalgarno. 1998. "The Deuterium Chemistry of the Early Universe." The Astrophysical Journal 509 (1): 1-10. https://doi.org/10.1086/306473.

\section{Permanent link}

http://nrs.harvard.edu/urn-3:HUL.InstRepos:41397387

\section{Terms of Use}

This article was downloaded from Harvard University's DASH repository, and is made available under the terms and conditions applicable to Other Posted Material, as set forth at http:// nrs.harvard.edu/urn-3:HUL.InstRepos:dash.current.terms-of-use\#LAA

\section{Share Your Story}

The Harvard community has made this article openly available.

Please share how this access benefits you. Submit a story.

Accessibility 
The AstrophysiCAL Journal, 509:1-10, 1998 December 10

(C) 1998. The American Astronomical Society. All rights reserved. Printed in U.S.A.

\title{
THE DEUTERIUM CHEMISTRY OF THE EARLY UNIVERSE
}

\author{
P. C. Stancil, ${ }^{1,2}$ S. Lepp, ${ }^{3}$ AND A. Dalgarno ${ }^{4}$ \\ Received 1998 March 2; accepted 1998 July 16
}

\begin{abstract}
The chemistry of deuterium, as well as that of hydrogen and helium, in the postrecombination era of the expanding early universe is presented. A thorough survey of all potentially important gas-phase reactions involving the primordial elements produced in the Big Bang, with a particular emphasis on deuterium, is given. The reaction set, consisting of 144 processes, is used in a nonequilibrium chemistry model to follow the production of primordial molecules in the postrecombination era. It is found that significant deuterium fractionation occurs for $\mathrm{HD}^{+}, \mathrm{HD}$, and $\mathrm{H}_{2} \mathrm{D}^{+}$, while the abundance of $\mathrm{D}^{+}$is reduced compared to the proton abundance. Even with the enhanced fractionation of $\mathrm{H}_{2} \mathrm{D}^{+}$, its abundance is predicted to be too small to cause any interesting cosmological consequences, such as possible attenuation of spatial anisotropies in the cosmic background radiation field, detections of the epochs of reionization and reheating, or constraints on the primordial deuterium abundance. HD, being the second most abundant primordial molecule after $\mathrm{H}_{2}$, may play a role in subsequent structure formation because of its cooling radiation.
\end{abstract}

Subject headings: cosmic microwave background - early universe - molecular processes nuclear reactions, nucleosynthesis, abundances

\section{INTRODUCTION}

Deuterium at early epochs has attracted renewed interest as a consequence of the controversy regarding its observed fractional abundance in high-redshift Ly $\alpha$ clouds (Songaila et al. 1994; Carswell et al. 1994, 1996; Tytler, Fan, \& Burles 1996; Burles \& Tytler 1996). Further, Dubrovich (1993) has suggested that primordial $\mathrm{H}_{2} \mathrm{D}^{+}$, which has a significant dipole moment $(\sim 0.6$ Debye), may be detected through its effect on the cosmic background radiation (CBR) field. If $\mathrm{H}_{2} \mathrm{D}^{+}$is sufficiently abundant, Thomson scattering of CBR photons may provide a partial attenuation of CBR spatial anisotropies. Finally, there remains optimism that HD may be observed in the first collapsing objects and that it may play a role in formation of these objects via its radiative cooling. It is important, therefore, to ensure that the deuterium chemistry is soundly established.

Early universe chemistry has been previously investigated by Lepp \& Shull (1983); Dalgarno \& Lepp (1987); Latter (1989); Puy et al. (1993); Palla, Galli, \& Silk (1995); Stancil, Lepp, \& Dalgarno (1996); Abel et al. (1997); Bougleux \& Galli (1997); and Galli \& Palla (1998; hereafter GP), while Dalgarno \& Lepp (1984), Pineau des Forêts, Roueff, \& Flower (1989), Rodgers \& Millar (1996), and others have discussed the chemistry of deuterium in the interstellar medium. We improve upon the previous work by constructing a comprehensive chemistry. Fractional abundances of $e^{-}, \mathrm{H}, \mathrm{H}^{+}, \mathrm{H}^{-}, \mathrm{D}, \mathrm{D}^{+}, \mathrm{D}^{-}, \mathrm{He}, \mathrm{He}^{+}, \mathrm{Li}, \mathrm{Li}^{+}, \mathrm{Li}^{-}, \mathrm{H}_{2}, \mathrm{H}_{2}^{+}$, $\mathrm{HD}, \mathrm{HD}^{+}, \mathrm{He}_{2}^{+}, \mathrm{HeH}^{+}, \mathrm{HeD}^{+}, \mathrm{H}_{3}^{+}, \mathrm{H}_{2} \mathrm{D}^{+}, \mathrm{LiH}$, and $\mathrm{LiH}^{+}$ are calculated for redshifts $z$ between 4000 and 10 in an expanding universe. 144 reactions are included with their rate coefficients taken from the most recent theoretical or experimental evaluations. Enhancements to molecule for-

\footnotetext{
${ }^{1}$ Physics Division, Oak Ridge National Laboratory, P.O. Box 2008, Oak Ridge, TN 37831-6372; stancil@mail.phy.ornl.gov.

${ }^{2}$ Eugene P. Wigner Fellow.

${ }^{3}$ W. M. Keck Laboratory for Computational Physics, Department of Physics, University of Nevada, Las Vegas, NV 89154-4002; lepp@physics.unlv.edu.

${ }^{4}$ Harvard-Smithsonian Center for Astrophysics, 60 Garden Street, Cambridge, MA 02138; adalgarno@cfa.harvard.edu.
}

mation through stimulated radiative association are included. The recombination-era gas-phase chemistry is discussed in $\S 2$, while $\S 3$ presents the adopted cosmological models. The results of atomic and molecular abundance calculations are presented in $\S 4$ with their cosmological implications discussed in $\S 5$.

\section{EARLY UNIVERSE CHEMISTRY}

After the epoch of nucleosynthesis, the universe was saturated with a high-temperature CBR field delaying both electronic and chemical bonding. As the universe expanded, it cooled, allowing for the formation in chronological order of $\mathrm{Li}^{2+}, \mathrm{He}^{+}, \mathrm{Li}^{+}, \mathrm{He}, \mathrm{D}, \mathrm{H}$, and $\mathrm{Li}$ by radiative recombination and $\mathrm{D}^{-}, \mathrm{H}^{-}$, and $\mathrm{Li}^{-}$through radiative attachment. With the formation of neutral helium, the universe was poised for the appearance of one of the first chemical bonds in $\mathrm{He}_{2}^{+}$by the radiative association process $(\mathrm{T} 1.98)^{5}$

$$
\mathrm{He}^{+}+\mathrm{He} \rightarrow \mathrm{He}_{2}^{+}+v .
$$

Its abundance was severely limited by the photodissociation reaction (T1.99) and dissociative recombination (T1.100). The formation of $\mathrm{He}_{2}^{+}$was later hindered by the removal of $\mathrm{He}^{+}$by radiative recombination (T1.17). As such, while it was the first molecule to form, it never reached sufficient abundances to be of interest. $\mathrm{LiHe}^{+}$was also produced and destroyed through similar processes (Dalgarno \& Fox 1994) but was even less abundant.

Following the production of neutral $\mathrm{He}$, the molecular ions $\mathrm{HeH}^{+}$and $\mathrm{HeD}^{+}$were formed by the radiative association processes (T1.37)

$$
\mathrm{He}+\mathrm{H}^{+} \rightarrow \mathrm{HeH}^{+}+v
$$

and (T1.70), respectively. Because these reactions proceed within the ground electronic state of the molecule, the frequency $v$ of the emitted radiation is comparable to that of CBR frequencies $v_{r}$. This suggests (V. K. Dubrovich 1996, private communication) that molecule formation could be

\footnotetext{
${ }^{5}$ The notation (T1.x) refers to process $(x)$ of Table 1.
} 
enhanced by the stimulated processes (T1.38)

$$
\mathrm{He}+\mathrm{H}^{+}+v_{r} \rightarrow \mathrm{HeH}^{+}+v+v_{r}
$$

and (T1.71). The effect was investigated by Stancil \& Dalgarno (1997a) and Zygelman, Stancil, \& Dalgarno (1998) and found to only increase molecular abundances by $\sim 20 \%-30 \%$.

With the appearance of neutral $\mathrm{H}$ and $\mathrm{D}$, reactions (T1.40) and (T1.69) produce additional $\mathrm{HeH}^{+}$and $\mathrm{HeD}^{+}$. The helium hydride ions are destroyed by photodissociation (T1.39) and (T1.72), by dissociative recombination (T1.43) and (T1.77), and by reactions with $\mathrm{H}$ (T1.41) and (T1.75). Electronic photodissociation reactions such as

$$
\mathrm{HeH}^{+}+v \rightarrow \mathrm{He}^{+}+\mathrm{H}
$$

are not important, because UV radiation is insignificant until after the first stars are created. The molecular ion $\mathrm{HeH}^{+}$can also be formed by the reaction of vibrationally excited $\mathrm{H}_{2}^{+}$with $\mathrm{He}$ (T1.42):

$$
\mathrm{H}_{2}^{+}(v \geq 2)+\mathrm{He} \rightarrow \mathrm{HeH}^{+}+\mathrm{H} .
$$

As the universe cooled further, the stage was set for the beginning of chemistry with the formation of the first neutral molecules. Since dust grains had yet to form, $\mathrm{H}_{2}$ could only be formed through the gas-phase $\mathrm{H}_{2}^{+}$and $\mathrm{H}^{-}$ sequences: radiative association (T1.27) followed by the charge exchange reaction (T1.31)

$$
\mathrm{H}_{2}^{+}+\mathrm{H} \rightarrow \mathrm{H}_{2}+\mathrm{H}^{+}
$$

and radiative attachment (T1.3)

$$
\mathrm{H}+e^{-} \rightarrow \mathrm{H}^{-}+v,
$$

followed by associative detachment (T1.36)

$$
\mathrm{H}^{-}+\mathrm{H} \rightarrow \mathrm{H}_{2}+e^{-} .
$$

HD was formed by the similar reactions (T1.51), (T1.53), (T1.58), (T1.10), (T1.67), and (T1.68). The sequence (T1.8)

$$
\mathrm{H}^{+}+\mathrm{D} \rightarrow \mathrm{H}+\mathrm{D}^{+},
$$

followed by (T1.63)

$$
\mathrm{H}_{2}+\mathrm{D}^{+} \rightarrow \mathrm{HD}+\mathrm{H}^{+} \text {, }
$$

which is the major source of HD in diffuse interstellar clouds (Dalgarno, Weisheit, \& Black 1973), may produce additional HD in the early universe, while the radiative association reaction (T1.61)

$$
\mathrm{H}+\mathrm{D} \rightarrow \mathrm{HD}+v
$$

(Stancil \& Dalgarno 1997b) gives only a minor contribution.

$\mathrm{H}_{3}^{+}$, an important ion in interstellar chemistry, is formed in the early universe by the hydrogen abstraction reaction (T1.44)

$$
\mathrm{H}_{2}^{+}+\mathrm{H}_{2} \rightarrow \mathrm{H}_{3}^{+}+\mathrm{H},
$$

but it is readily removed via dissociative recombination (T1.48)

$$
\mathrm{H}_{3}^{+}+e^{-} \rightarrow \mathrm{H}_{2}+\mathrm{H}
$$

and (T1.49)

$$
\mathrm{H}_{3}^{+}+e^{-} \rightarrow \mathrm{H}+\mathrm{H}+\mathrm{H} .
$$

Collisions with D (T1.87)

$$
\mathrm{D}+\mathrm{H}_{3}^{+} \rightarrow \mathrm{H}_{2} \mathrm{D}^{+}+\mathrm{H}
$$

can deplete the $\mathrm{H}_{3}^{+}$abundance and form $\mathrm{H}_{2} \mathrm{D}^{+}$. The deuterated molecular ion $\mathrm{H}_{2} \mathrm{D}^{+}$is also produced by (T1.86)

$$
\mathrm{HD}^{+}+\mathrm{H}_{2} \rightarrow \mathrm{H}_{2} \mathrm{D}^{+}+\mathrm{H}
$$

and (T1.88)

$$
\mathrm{HD}+\mathrm{H}_{2}^{+} \rightarrow \mathrm{H}_{2} \mathrm{D}^{+}+\mathrm{H}
$$

and destroyed by dissociative recombination (T1.83)(T1.85)

$$
\begin{gathered}
\mathrm{H}_{2} \mathrm{D}^{+}+e^{-} \rightarrow \mathrm{H}+\mathrm{H}+\mathrm{D}, \\
\mathrm{H}_{2} \mathrm{D}^{+}+e^{-} \rightarrow \mathrm{H}_{2}+\mathrm{D},
\end{gathered}
$$

and

$$
\mathrm{H}_{2} \mathrm{D}^{+}+e^{-} \rightarrow \mathrm{HD}+\mathrm{H} .
$$

The presently adopted reaction rate coefficients $\alpha(T)$ are listed in Table 1 . They are taken from the most recent calculations or experiments available; others are deduced from experimental or theoretical studies on related systems, while the remainder are estimates and are subject to considerable uncertainty. If no data exist for a reaction involving deuterium, we adopt a rate coefficient equal to the analogous hydrogen reaction but scaled by the ratio of the collision system reduced-mass $\mu$, assuming the rate coefficients to have the form $\alpha(T) \propto T^{m} \propto 1 / \mu^{m}$, where $m$ is some power. The sources of the data are given in the table, and the reactions are generally listed for the exothermic direction.

\section{COSMOLOGICAL MODELS}

To estimate the particle abundances in the expanding universe, we take into account the chemical reactions listed in Table 1 and the lithium processes tabulated in Stancil et al. (1996) ${ }^{6}$. The corresponding chemical rate equations form a set of coupled stiff differential equations for the particle densities $n(x)$ of the form

$$
\frac{d n(x)}{d t}=\alpha_{\text {form }}\left(T_{m}\right) n(y) n(w)-\zeta_{\text {dest }}\left(T_{r}\right) n(x)-\alpha_{\text {dest }}\left(T_{m}\right) n(x) n(u),
$$

which depend upon the total density and the temperature, and which must be integrated in time $t$. The photodestruction rate $\zeta_{\text {dest }}$ is obtained by detailed balance using the appropriate equilibrium constant and the corresponding formation rate $\alpha_{\text {form }}$. Partition functions are taken from Irwin (1981), while the equilibrium constants are given by Sauval \& Tatum (1994), except for $\mathrm{HeH}^{+}$, which was taken from Gaur \& Tripathi (1985).

For the recombination epoch we assume that the hydrogen density is given by

$$
n_{\mathrm{H}}=1.123 \times 10^{-5}(1-Y) \Omega_{b} h^{2}(1+z)^{3} \mathrm{~cm}^{-3}
$$

\footnotetext{
${ }^{6}$ See Stancil \& Dalgarno (1997a, 1998) and Stancil \& Zygelman (1996) for some improvements to the lithium chemistry. Additionally, the radiative association reaction $\mathrm{Li}+\mathrm{H} \rightarrow \mathrm{LiH}+v$ should have the form $3.74 \times 10^{-20}(T / 300)^{0.11} \exp (-T / 2000) \mathrm{cm}^{3} \mathrm{~s}^{-1}$ for $T<380 \mathrm{~K}$, and the mutual neutralization reactions $\mathrm{Li}^{+}+\mathrm{H}^{-} \rightarrow \mathrm{Li}+\mathrm{H}$ and $\mathrm{Li}^{-}+\mathrm{H}^{+} \rightarrow \mathrm{Li}+\mathrm{H}$ are better represented by the fit $1.57 \times 10^{-7}(\mathrm{~T} /$ $300)^{-0.49} \exp (T / 11800) \mathrm{cm}^{3} \mathrm{~s}^{-1}$.
} 
TABLE 1

Gas-Phase Reactions and Their Rate Coefficients

\begin{tabular}{|c|c|c|c|c|}
\hline Reaction & $a_{1}\left(\mathrm{~cm}^{3} \mathrm{~s}^{-1}\right)$ & $a_{2}$ & $a_{3}(\mathrm{~K})$ & Notes \\
\hline (1) $\mathrm{H}^{+}+e^{-} \rightarrow \mathrm{H}+v \ldots \ldots$ & $3.6 \mathrm{E}-12^{\mathrm{a}}$ & -0.75 & $\cdots$ & 1 \\
\hline $\begin{array}{l}\text { (2) } \mathrm{H}+v \rightarrow \mathrm{H}^{+}+e^{-} \\
\text {(3) } \mathrm{H}+e^{-} \rightarrow \mathrm{H}^{-}+v \ldots \ldots \ldots \ldots \ldots \ldots \ldots \ldots \ldots \ldots \ldots \ldots \ldots \ldots \ldots \ldots\end{array}$ & $3.0 \mathrm{E}-16$ & 0.95 & 9320 & $\begin{array}{l}2 \\
3\end{array}$ \\
\hline 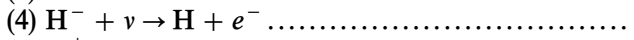 & & & $\ldots$ & 2 \\
\hline (5) $\mathrm{H}^{+}+\mathrm{H}^{-} \rightarrow \mathrm{H}+\mathrm{H} \ldots \ldots$ & $4.0 \mathrm{E}-8$ & -0.50 & $\ldots$ & 4 \\
\hline (6) $\mathrm{D}^{+}+e^{-} \rightarrow \mathrm{D}+v \ldots \ldots$ & $3.6 \mathrm{E}-12$ & -0.75 & $\ldots$ & 1 \\
\hline (7) $\mathrm{D}+v \rightarrow \mathrm{D}^{+}+e^{-} \ldots$ & & $\ldots$ & & 2 \\
\hline (8) $\mathrm{H}^{+}+\mathrm{D} \rightarrow \mathrm{H}+\mathrm{D}^{+}$. & $1.0 \mathrm{E}-9$ & $\ldots$ & $41^{\mathrm{b}}$ & 5 \\
\hline (9) $\mathrm{D}^{+}+\mathrm{H} \rightarrow \mathrm{D}+\mathrm{H}^{+} \ldots$ & $1.0 \mathrm{E}-9$ & & & 5 \\
\hline (10) $\mathrm{D}+e^{-} \rightarrow \mathrm{D}^{-}+v \ldots \ldots$ & $3.0 \mathrm{E}-16$ & 0.95 & 9320 & 6 \\
\hline (11) $\mathrm{D}^{-}+v \rightarrow \mathrm{D}+e^{-} \ldots \ldots \ldots$ & & & $\ldots$ & 2 \\
\hline (12) $\mathrm{D}^{+}+\mathrm{D}^{-} \rightarrow \mathrm{D}+\mathrm{D} \ldots \ldots$ & $5.7 \mathrm{E}-8$ & -0.50 & $\ldots$ & 7 \\
\hline 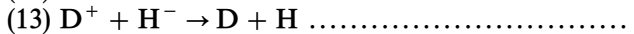 & $4.6 \mathrm{E}-8$ & -0.50 & $\ldots$ & 7 \\
\hline (14) $\mathrm{H}^{+}+\mathrm{D}^{-} \rightarrow \mathrm{D}+\mathrm{H} \ldots \ldots \ldots \ldots$ & $4.6 \mathrm{E}-8$ & -0.50 & $\ldots$ & 7 \\
\hline (15) $\mathrm{H}^{-}+\mathrm{D} \rightarrow \mathrm{H}+\mathrm{D}^{-} \ldots \ldots$ & $6.4 \mathrm{E}-9$ & 0.41 & $\ldots$ & 8 \\
\hline (16) $\mathrm{D}^{-}+\mathrm{H} \rightarrow \mathrm{D}+\mathrm{H}^{-} \ldots$ & $6.4 \mathrm{E}-9$ & 0.41 & $\ldots$ & 8 \\
\hline (17) $\mathrm{He}^{+}+e^{-} \rightarrow \mathrm{He}+v \ldots \ldots \ldots \ldots$ & $4.51 \mathrm{E}-12$ & -0.67 & $\ldots$ & 9 \\
\hline (18) $\mathrm{He}+v \rightarrow \mathrm{He}^{+}+e^{-} \ldots \ldots \ldots$ & & & & 2 \\
\hline (19) $\mathrm{He}^{+}+\mathrm{H} \rightarrow \mathrm{He}+\mathrm{H}^{+}+v$. & $1.20 \mathrm{E}-15$ & 0.25 & & 10 \\
\hline (20) $\mathrm{He}^{+}+\mathrm{H}^{-} \rightarrow \mathrm{He}+\mathrm{H} \ldots \ldots \ldots .$. & $2.32 \mathrm{E}-7$ & -0.52 & -22400 & 11 \\
\hline (21) $\mathrm{He}^{+}+\mathrm{D} \rightarrow \mathrm{He}+\mathrm{D}^{+}+v \ldots \ldots \ldots \ldots \ldots \ldots \ldots$ & $1.1 \mathrm{E}-15$ & 0.25 & & 7 \\
\hline (22) $\mathrm{He}^{+}+\mathrm{D}^{-} \rightarrow \mathrm{He}+\mathrm{D} \ldots \ldots \ldots \ldots \ldots \ldots \ldots$ & $3.03 \mathrm{E}-7$ & -0.52 & -22400 & 7 \\
\hline (23) $\mathrm{Li}+\mathrm{D}^{+} \rightarrow \mathrm{Li}^{+}+\mathrm{D}+v \ldots \ldots \ldots \ldots \ldots \ldots \ldots \ldots \ldots \ldots \ldots \ldots \ldots \ldots$ & $1.1 \mathrm{E}-13$ & $-5.1 \mathrm{E}-2$ & 282000 & 12 \\
\hline (24) $\mathrm{Li}^{+}+\mathrm{D}^{-} \rightarrow \mathrm{Li}+\mathrm{D} \ldots \ldots \ldots$ & $2.06 \mathrm{E}-7$ & -0.50 & -18300 & 11 \\
\hline (25) $\mathrm{Li}+\mathrm{D}^{+} \rightarrow \mathrm{Li}^{+}+\mathrm{D} \ldots \ldots \ldots$ & $8.0 \mathrm{E}-22$ & 6.80 & 1800 & 13 \\
\hline (26) $\mathrm{Li}^{-}+\mathrm{D}^{+} \rightarrow \mathrm{Li}+\mathrm{D} \ldots \ldots \ldots \ldots \ldots$ & $2.06 \mathrm{E}-7$ & -0.50 & -18300 & 14 \\
\hline 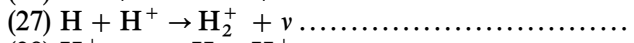 & $6.0 \mathrm{E}-19$ & 1.8 & $-20^{\mathrm{b}}$ & 15 \\
\hline (28) $\mathrm{H}_{2}^{+}+v \rightarrow \mathrm{H}+\mathrm{H}^{+} \ldots \ldots \ldots$ & & & .. & 2 \\
\hline (29) $\mathrm{H}^{+}+\mathrm{H}^{-} \rightarrow \mathrm{H}_{2}^{+}+e^{-}$ & $1.0 \mathrm{E}-9$ & -0.4 & $\cdots$ & 16 \\
\hline (30) $\mathrm{H}_{2}^{+}+e^{-} \rightarrow \mathrm{H}+\mathrm{H} \ldots \ldots$ & $1.2 \mathrm{E}-8$ & -0.4 & $\ldots$ & 17 \\
\hline (31) $\mathrm{H}_{2}^{+}+\mathrm{H} \rightarrow \mathrm{H}_{2}+\mathrm{H}^{+}$. & $6.4 \mathrm{E}-10$ & 0 & $\ldots$ & 18 \\
\hline (32) $\mathrm{H}_{2}^{+}+\mathrm{H}^{-} \rightarrow \mathrm{H}_{2}+\mathrm{H} \ldots \ldots$ & $1.4 \mathrm{E}-7$ & -0.5 & $\ldots$ & 4 \\
\hline (33) $\mathrm{H}_{2}^{+}+\mathrm{H}^{-} \rightarrow \mathrm{H}+\mathrm{H}+\mathrm{H} \ldots \ldots$ & $1.4 \mathrm{E}-7$ & -0.5 & $\ldots$ & 4 \\
\hline (34) $\mathrm{H}_{2}+\mathrm{He}^{+} \rightarrow \mathrm{H}_{2}^{+}+\mathrm{He}+v \ldots \ldots$ & $7.2 \mathrm{E}-15$ & 0 & $\ldots$ & 19 \\
\hline (35) $\mathrm{H}_{2}+\mathrm{He}^{+} \rightarrow \mathrm{He}+\mathrm{H}+\mathrm{H}^{+} \ldots \ldots \ldots \ldots \ldots \ldots \ldots$ & $3.7 \mathrm{E}-14$ & 0 & $35^{\mathrm{b}}$ & 19 \\
\hline (36) $\mathrm{H}+\mathrm{H}^{-} \rightarrow \mathrm{H}_{2}+e^{-} \ldots \ldots \ldots \ldots$ & $1.5 \mathrm{E}-9$ & -0.1 & & 20 \\
\hline (37) $\mathrm{He}+\mathrm{H}^{+} \rightarrow \mathrm{HeH}^{+}+v \ldots \ldots$ & $8.0 \mathrm{E}-20$ & -0.24 & 4000 & 21 \\
\hline (38) $\mathrm{He}+\mathrm{H}^{+}+v_{r} \rightarrow \mathrm{HeH}^{+}+v+v_{r} \ldots \ldots \ldots \ldots \ldots \ldots$ & $1.5 \mathrm{E}-20$ & 0 & $200^{\mathrm{b}}$ & 22 \\
\hline (39) $\mathrm{HeH}^{+}+v \rightarrow \mathrm{He}+\mathrm{H}^{+} \ldots \ldots \ldots \ldots \ldots$ & & & & 2 \\
\hline (40) $\mathrm{He}^{+}+\mathrm{H} \rightarrow \mathrm{HeH}^{+}+v \ldots \ldots$ & $4.16 \mathrm{E}-16$ & -0.37 & 87600 & 23 \\
\hline (41) $\mathrm{HeH}^{+}+\mathrm{H} \rightarrow \mathrm{H}_{2}^{+}+\mathrm{He} \ldots \ldots$. & $1.04 \mathrm{E}-9$ & 0.13 & 33100 & 24 \\
\hline (42) $\mathrm{He}+\mathrm{H}_{2}^{+} \rightarrow \mathrm{HeH}^{+}+\mathrm{H} \ldots \ldots$ & $3.0 \mathrm{E}-10$ & 0 & $6720^{\mathrm{b}}$ & 25 \\
\hline (43) $\mathrm{HeH}^{+}+e^{-} \rightarrow \mathrm{He}+\mathrm{H} \ldots \ldots \ldots \ldots \ldots \ldots \ldots \ldots \ldots$ & $3.0 \mathrm{E}-8$ & -0.47 & $\ldots$ & 26 \\
\hline (44) $\mathrm{H}_{2}^{+}+\mathrm{H}_{2} \rightarrow \mathrm{H}_{3}^{+}+\mathrm{H} \ldots \ldots \ldots \ldots \ldots \ldots \ldots \ldots \ldots \ldots$ & $2.24 \mathrm{E}-9$ & $4.2 \mathrm{E}-2$ & 46600 & 24 \\
\hline (45) $\mathrm{HeH}^{+}+\mathrm{H}_{2} \rightarrow \mathrm{H}_{3}^{+}+\mathrm{He} \ldots \ldots \ldots \ldots \ldots \ldots \ldots \ldots$ & $1.53 \mathrm{E}-9$ & 0.24 & 14800 & 24 \\
\hline (46) $\mathrm{H}_{2}+\mathrm{H}^{+} \rightarrow \mathrm{H}_{3}^{+}+v \ldots \ldots \ldots \ldots \ldots \ldots \ldots \ldots \ldots \ldots$ & $1.0 \mathrm{E}-20$ & & & 8 \\
\hline (47) $\mathrm{H}_{2}^{+}+\mathbf{H} \rightarrow \mathbf{H}_{3}^{+}+v \ldots \ldots \ldots \ldots$ & $1.5 \mathrm{E}-17$ & 1.8 & $-20^{b}$ & 8 \\
\hline 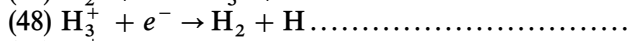 & $4.0 \mathrm{E}-8$ & -0.5 & $\ldots$ & 27 \\
\hline (49) $\mathrm{H}_{3}^{+}+e^{-} \rightarrow \mathrm{H}+\mathrm{H}+\mathrm{H} \ldots \ldots$ & $1.6 \mathrm{E}-7$ & -0.5 & $\ldots$ & 27 \\
\hline (50) $\mathrm{H}_{3}^{+}+\mathrm{H}^{-} \rightarrow \mathrm{H}_{2}+\mathrm{H}+\mathrm{H} \ldots \ldots$. & $2.3 \mathrm{E}-7$ & -0.5 & $\ldots$ & 8 \\
\hline 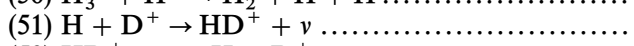 & $3.9 \mathrm{E}-19$ & 1.8 & $-20^{\mathrm{b}}$ & 28 \\
\hline (52) $\mathrm{HD}^{+}+v \rightarrow \mathrm{H}+\mathrm{D}^{+} \ldots \ldots \ldots \ldots \ldots \ldots \ldots \ldots \ldots$ & & & & 2 \\
\hline (53) $\mathrm{D}+\mathrm{H}^{+} \rightarrow \mathrm{HD}^{+}+v \ldots \ldots \ldots \ldots \ldots \ldots \ldots \ldots \ldots \ldots$ & $3.9 \mathrm{E}-19$ & 1.8 & $-20^{\mathrm{b}}$ & 28 \\
\hline (54) $\mathrm{HD}^{+}+v \rightarrow \mathrm{D}+\mathrm{H}^{+} \ldots \ldots \ldots \ldots \ldots \ldots \ldots \ldots \ldots \ldots \ldots \ldots \ldots \ldots \ldots$ & & & & 2 \\
\hline (55) $\mathrm{H}_{2}^{+}+\mathrm{D} \rightarrow \mathrm{HD}^{+}+\mathrm{H} \ldots \ldots \ldots \ldots \ldots \ldots \ldots \ldots \ldots \ldots$ & $1.07 \mathrm{E}-9$ & $6.2 \mathrm{E}-2$ & 41400 & 24 \\
\hline (56) $\mathrm{H}_{2}^{+}+\mathrm{D} \rightarrow \mathrm{H}_{2}+\mathrm{D}^{+} \ldots \ldots \ldots \ldots \ldots \ldots \ldots \ldots \ldots$ & $6.4 \mathrm{E}-10$ & 0 & $\ldots$ & 6 \\
\hline 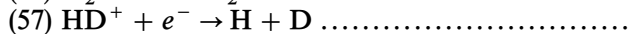 & $3.4 \mathrm{E}-9$ & -0.4 & $\ldots$ & 29 \\
\hline (58) $\mathrm{HD}^{+}+\mathrm{H} \rightarrow \mathrm{HD}+\mathrm{H}^{+} \ldots \ldots \ldots \ldots \ldots \ldots \ldots \ldots \ldots$ & $6.4 \mathrm{E}-10$ & 0 & $\ldots$ & 6 \\
\hline 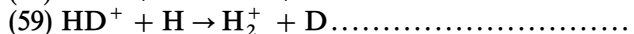 & $1.0 \mathrm{E}-9$ & 0 & $154^{\mathrm{b}}$ & 8 \\
\hline 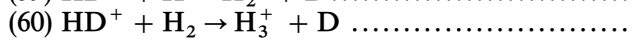 & $1.05 \mathrm{E}-9$ & 0 & & 5 \\
\hline 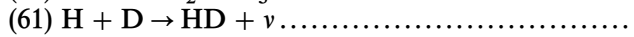 & $1.05 \mathrm{E}-26$ & 0.09 & 7000 & 30 \\
\hline (62) $\mathrm{H}+\mathrm{D}+v_{r} \rightarrow \mathrm{HD}+v+v_{r} \ldots$ & $2.0 \mathrm{E}-27$ & 0 & $400^{\mathrm{b}}$ & 30 \\
\hline (63) $\mathrm{H}_{2}+\mathrm{D}^{+} \rightarrow \mathrm{HD}+\mathrm{H}^{+} \ldots \ldots \ldots \ldots$ & $2.1 \mathrm{E}-9$ & 0 & .. & 31 \\
\hline (64) $\mathrm{HD}+\mathrm{H}^{+} \rightarrow \mathrm{H}_{2}+\mathrm{D}^{+}$.. & $1.0 \mathrm{E}-9$ & 0 & $457^{\mathrm{b}}$ & 31 \\
\hline (65) $\mathrm{H}_{2}+\mathrm{D} \rightarrow \mathrm{HD}+\mathrm{H} \ldots \ldots \ldots \ldots \ldots \ldots \ldots \ldots \ldots$ & $7.5 \mathrm{E}-11$ & 0 & $3820^{\mathrm{b}}$ & 32 \\
\hline (66) $\mathrm{HD}+\mathrm{H} \rightarrow \mathrm{H}_{2}+\mathrm{D} \ldots \ldots \ldots \ldots \ldots \ldots \ldots \ldots \ldots \ldots \ldots$ & $7.5 \mathrm{E}-11$ & 0 & $4240^{b}$ & 32 \\
\hline (67) $\mathrm{D}+\mathrm{H}^{-} \rightarrow \mathrm{HD}+e^{-} \ldots \ldots \ldots \ldots \ldots \ldots \ldots \ldots \ldots \ldots \ldots \ldots \ldots \ldots \ldots$ & $1.5 \mathrm{E}-9$ & -0.1 & $\ldots$ & 7 \\
\hline (68) $\mathrm{D}^{-}+\mathrm{H} \rightarrow \mathrm{HD}+e^{-} \ldots \ldots \ldots \ldots \ldots \ldots$ & $1.5 \mathrm{E}-9$ & -0.1 & $\ldots$ & 7 \\
\hline (69) $\mathrm{He}^{+}+\mathrm{D} \rightarrow \mathrm{HeD}^{+}+v \ldots \ldots \ldots \ldots \ldots \ldots \ldots \ldots \ldots \ldots \ldots \ldots \ldots \ldots \ldots$ & $5.0 \mathrm{E}-16$ & -0.37 & 87600 & 7 \\
\hline (70) $\mathrm{He}+\mathrm{D}^{+} \rightarrow \mathrm{HeD}^{+}+v \ldots \ldots \ldots \ldots \ldots \ldots \ldots \ldots \ldots \ldots \ldots \ldots \ldots \ldots \ldots \ldots$ & $1.0 \mathrm{E}-19$ & -0.24 & 4000 & 7 \\
\hline (71) $\mathrm{He}+\mathrm{D}^{+}+v_{r} \rightarrow \mathrm{HeD}^{+}+v+v_{r} \ldots \ldots \ldots \ldots \ldots$ & $1.5 \mathrm{E}-21$ & 0 & $200^{\mathrm{b}}$ & 7 \\
\hline (72) $\mathrm{HeD}^{+}+v \rightarrow \mathrm{He}+\mathrm{D}^{+} \ldots \ldots \ldots$ & & & & 2 \\
\hline (73) $\mathrm{HeH}^{+}+\mathrm{D} \rightarrow \mathrm{HeD}^{+}+\mathrm{H}$ & $1.0 \mathrm{E}-9$ & 0 & & 8 \\
\hline
\end{tabular}


TABLE 1-Continued

\begin{tabular}{|c|c|c|c|c|}
\hline Reaction & $a_{1}\left(\mathrm{~cm}^{3} \mathrm{~s}^{-1}\right)$ & $a_{2}$ & $a_{3}(\mathrm{~K})$ & Notes \\
\hline (74) $\mathrm{HeD}^{+}+\mathrm{H} \rightarrow \mathrm{HeH}^{+}+\mathrm{D}$ & $8.0 \mathrm{E}-10$ & 0 & $468^{\mathrm{b}}$ & 8 \\
\hline (75) $\mathrm{HeD}^{+}+\mathrm{H} \rightarrow \mathrm{HD}^{+}+\mathrm{He}$. & $9.1 \mathrm{E}-10$ & 0.13 & 33100 & 6 \\
\hline (76) $\mathrm{HeH}^{+}+\mathrm{D} \rightarrow \mathrm{HD}^{+}+\mathrm{He}$. & $8.5 \mathrm{E}-10$ & 0.13 & 33100 & 6 \\
\hline (77) $\mathrm{HeD}^{+}+e^{-} \rightarrow \mathrm{He}+\mathrm{D} \ldots$ & $3.0 \mathrm{E}-8$ & -0.47 & $\ldots$ & 6 \\
\hline (78) $\mathrm{H}_{3}^{+}+\mathrm{HD} \rightarrow \mathrm{H}_{2} \mathrm{D}^{+}+\mathrm{H}_{2}$. & $1.4 \mathrm{E}-9$ & 0 & $\ldots$ & 31 \\
\hline (79) $\mathrm{H}_{2}+\mathrm{D}^{+} \rightarrow \mathrm{H}_{2} \mathrm{D}^{+}+v \ldots \ldots$ & $1.0 \mathrm{E}-20$ & $\ldots$ & $\ldots$ & 6 \\
\hline (80) $\mathrm{HD}+\mathrm{H}^{+} \rightarrow \mathrm{H}_{2} \mathrm{D}^{+}+v \ldots$ & $1.0 \mathrm{E}-20$ & $\ldots$ & & 6 \\
\hline (81) $\mathrm{H}_{2}^{+}+\mathrm{D} \rightarrow \mathrm{H}_{2} \mathrm{D}^{+}+v \ldots \ldots$ & $7.0 \mathrm{E}-18$ & 1.8 & $-20^{b}$ & 6 \\
\hline (82) $\mathrm{HD}^{+}+\mathrm{H} \rightarrow \mathrm{H}_{2} \mathrm{D}^{+}+v \ldots$ & $1.2 \mathrm{E}-17$ & 1.8 & $-20^{\mathrm{b}}$ & 6 \\
\hline (83) $\mathrm{H}_{2} \mathrm{D}^{+}+e^{-} \rightarrow \mathrm{H}+\mathrm{H}+\mathrm{D}$. & $4.38 \mathrm{E}-8$ & -0.5 & $\ldots$ & 33 \\
\hline (84) $\mathrm{H}_{2} \mathrm{D}^{+}+e^{-} \rightarrow \mathrm{H}_{2}+\mathrm{D} \ldots \ldots$ & $4.2 \mathrm{E}-9$ & -0.5 & $\ldots$ & 33 \\
\hline (85) $\mathrm{H}_{2} \mathrm{D}^{+}+e^{-} \rightarrow \mathrm{H}+\mathrm{HD} \ldots$ & $1.2 \mathrm{E}-8$ & -0.5 & $\ldots$ & 33 \\
\hline (86) $\mathrm{HD}^{+}+\mathrm{H}_{2} \rightarrow \mathrm{H}_{2} \mathrm{D}^{+}+\mathrm{H}$. & $1.05 \mathrm{E}-9$ & 0 & $\ldots$ & 5 \\
\hline (87) $\mathrm{H}_{3}^{+}+\mathrm{D} \rightarrow \mathrm{H}_{2} \mathrm{D}^{+}+\mathrm{H} \ldots \ldots$ & $1.0 \mathrm{E}-9$ & 0 & $\ldots$ & 34 \\
\hline (88) $\mathrm{HD}+\mathrm{H}_{2}^{+} \rightarrow \mathrm{H}_{2} \mathrm{D}^{+}+\mathrm{H} \ldots$ & $1.05 \mathrm{E}-9$ & 0 & $\ldots$ & 35 \\
\hline (89) $\mathrm{HD}+\mathrm{H}_{2}^{+} \rightarrow \mathrm{H}_{3}^{+}+\mathrm{D} \ldots$ & $1.05 \mathrm{E}-9$ & 0 & & 35 \\
\hline (90) $\mathrm{H}_{2} \mathrm{D}^{+}+\mathrm{H} \rightarrow \mathrm{H}_{3}^{+}+\mathrm{D} \ldots \ldots$ & $1.0 \mathrm{E}-9$ & 0 & $632^{\mathrm{b}}$ & 5 \\
\hline (91) $\mathrm{H}_{2} \mathrm{D}^{+}+\mathrm{H}_{2} \rightarrow \mathrm{H}_{3}^{+}+\mathrm{HD} \ldots \ldots$ & $1.7 \mathrm{E}-9$ & 0 & $150^{\mathrm{b}}$ & 31 \\
\hline (92) $\mathrm{HeD}^{+}+\mathrm{H}_{2} \rightarrow \mathrm{H}_{2} \mathrm{D}^{+}+\mathrm{He} \ldots$ & $1.24 \mathrm{E}-9$ & 0.24 & 14800 & 6 \\
\hline (93) $\mathrm{HeH}^{+}+\mathrm{HD} \rightarrow \mathrm{H}_{2} \mathrm{D}^{+}+\mathrm{He} \ldots$ & $1.20 \mathrm{E}-9$ & 0.24 & 14800 & 6 \\
\hline (94) $\mathrm{He}^{+}+\mathrm{HD} \rightarrow \mathrm{He}+\mathrm{H}^{+}+\mathrm{D}$. & $5.5 \mathrm{E}-14$ & -0.24 & $\ldots$ & 5 \\
\hline (95) $\mathrm{He}^{+}+\mathrm{HD} \rightarrow \mathrm{He}+\mathrm{H}+\mathrm{D}^{+}$ & $5.5 \mathrm{E}-14$ & -0.24 & & 5 \\
\hline (96) $\mathrm{He}+\mathrm{HD}^{+} \rightarrow \mathrm{HeH}^{+}+\mathrm{D} \ldots$ & $3.0 \mathrm{E}-10$ & 0 & $6720^{\mathrm{b}}$ & 6 \\
\hline (97) $\mathrm{He}+\mathrm{HD}^{+} \rightarrow \mathrm{HeD}^{+}+\mathrm{H}$. & $3.0 \mathrm{E}-10$ & 0 & $6720^{\mathrm{b}}$ & 6 \\
\hline (98) $\mathrm{He}+\mathrm{He}^{+} \rightarrow \mathrm{He}_{2}^{+}+v \ldots$. & $4.76 \mathrm{E}-20$ & 1.82 & $-29^{b}$ & 36 \\
\hline (99) $\mathrm{He}_{2}^{+}+v \rightarrow \mathrm{He}+\mathrm{He}^{+} \ldots$ & & & $\ldots$ & 2 \\
\hline (100) $\mathrm{He}_{2}^{+}+e^{-} \rightarrow \mathrm{He}+\mathrm{He} \ldots$ & $5.0 \mathrm{E}-10$ & -0.5 & .. & 37 \\
\hline (101) $\mathrm{He}_{2}^{+}+\mathrm{H} \rightarrow \mathrm{He}+\mathrm{He}+\mathrm{H}^{+}$. & $1.0 \mathrm{E}-9$ & 0 & & 38 \\
\hline (102) $\mathrm{LiH}^{+}+\mathrm{D} \rightarrow \mathrm{Li}+\mathrm{HD}^{+}$ & $9.0 \mathrm{E}-10$ & $\ldots$ & $66400^{\mathrm{b}}$ & 39 \\
\hline (103) $\mathrm{LiH}^{+}+\mathrm{D} \rightarrow \mathrm{Li}^{+}+\mathrm{HD}$ & $3.0 \mathrm{E}-10$ & $\ldots$ & & 39 \\
\hline (104) $\mathrm{LiH}^{+}+\mathrm{D} \rightarrow \mathrm{LiH}+\mathrm{D}^{+}$. & $1.0 \mathrm{E}-11$ & $\ldots$ & $67900^{\mathrm{b}}$ & 39 \\
\hline (105) $\mathrm{LiH}+\mathrm{D}^{+} \rightarrow \mathrm{LiH}^{+}+\mathrm{D}$ & $1.0 \mathrm{E}-9$ & $\ldots$ & $\ldots$ & 39 \\
\hline (106) $\mathrm{LiH}+\mathrm{D} \rightarrow \mathrm{Li}+\mathrm{HD} \ldots \ldots$ & $2.0 \mathrm{E}-11$ & $\ldots$ & $\ldots$ & 39 \\
\hline (107) $\mathrm{LiH}+\mathrm{D}^{+} \rightarrow \mathrm{Li}^{+}+\mathrm{HD}$ & $1.0 \mathrm{E}-9$ & $\cdots$ & . & 39 \\
\hline (108) $\mathrm{LiH}+\mathrm{D}^{+} \rightarrow \mathrm{Li}+\mathrm{HD}^{+} .$. & $1.0 \mathrm{E}-9$ & ( & & 39 \\
\hline 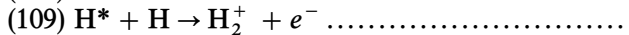 & $6.8 \mathrm{E}-12$ & 0.61 & $13000^{\mathrm{b}}$ & 40 \\
\hline$(110) \mathrm{H}^{*}+\mathrm{H} \rightarrow \mathrm{H}_{2}+v \ldots \ldots \ldots \ldots \ldots \ldots \ldots \ldots \ldots \ldots \ldots \ldots \ldots \ldots \ldots$ & $2.09 \mathrm{E}-14$ & 0.24 & 37800 & 41 \\
\hline (111) $\mathrm{H}^{*}+\mathrm{H}_{2} \rightarrow \mathrm{H}_{3}^{+}+e^{-} \ldots \ldots \ldots \ldots \ldots \ldots \ldots \ldots$ & $4.7 \mathrm{E}-9$ & -0.5 & $\ldots$ & 42 \\
\hline (112) $\mathrm{H}^{*}+\mathrm{H}_{2} \rightarrow \mathrm{H}+\mathrm{H}+\mathrm{H} \ldots \ldots \ldots \ldots \ldots \ldots \ldots$ & $2.5 \mathrm{E}-11$ & $\ldots$ & & 43 \\
\hline (113) $\mathrm{H}^{*}+\mathrm{D} \rightarrow \mathrm{HD}^{+}+e^{-} \ldots \ldots \ldots \ldots \ldots \ldots \ldots \ldots$ & $5.7 \mathrm{E}-12$ & 0.61 & $13000^{\mathrm{b}}$ & 7 \\
\hline (114) $\mathrm{D}^{*}+\mathrm{H} \rightarrow \mathrm{HD}^{+}+e^{-} \ldots$ & $5.7 \mathrm{E}-12$ & 0.61 & $13000^{\mathrm{b}}$ & 7 \\
\hline (115) $\mathrm{H}^{*}+\mathrm{D} \rightarrow \mathrm{HD}+v \ldots \ldots \ldots$ & $2.0 \mathrm{E}-14$ & 0.24 & 37800 & 7 \\
\hline 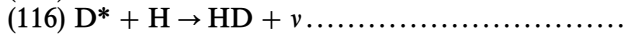 & $2.0 \mathrm{E}-14$ & 0.24 & 37800 & 7 \\
\hline (117) $\mathrm{H}^{*}+\mathrm{HD} \rightarrow \mathrm{H}_{2} \mathrm{D}^{+}+e^{-} \ldots \ldots \ldots \ldots \ldots \ldots \ldots$ & $5.4 \mathrm{E}-9$ & -0.5 & $\ldots$ & 7 \\
\hline (118) $\mathrm{D}^{*}+\mathrm{H}_{2} \rightarrow \mathrm{H}_{2} \mathrm{D}^{+}+e^{-} \ldots \ldots \ldots \ldots \ldots \ldots \ldots$ & $5.8 \mathrm{E}-9$ & -0.5 & 0 & 7 \\
\hline (119) $\mathrm{H}^{*}+\mathrm{HD} \rightarrow \mathrm{H}+\mathrm{H}+\mathrm{D} \ldots \ldots \ldots \ldots \ldots \ldots \ldots \ldots \ldots \ldots$ & $2.5 \mathrm{E}-11$ & $\ldots$ & $\cdots$ & 6 \\
\hline$(120) \mathrm{D}^{*}+\mathrm{H}_{2} \rightarrow \mathrm{H}+\mathrm{H}+\mathrm{D} \ldots \ldots \ldots \ldots \ldots \ldots \ldots \ldots \ldots \ldots \ldots$ & $2.5 \mathrm{E}-11$ & $\ldots$ & $\ldots$ & 6 \\
\hline
\end{tabular}

NoTE. -The rate coefficients fits are given by the relation $\alpha=a_{1}(T / 300)^{a_{2}} \exp \left(-T / a_{3}\right)$.

a The notation $3.6 \mathrm{E}-12$ corresponds to $3.6 \times 10^{-12}$.

${ }^{\mathrm{b}}$ For the indicated reactions the exponential term in the rate relation has the form $\exp \left(-a_{3} / T\right)$.

REFERENCES.-(1) Hollenbach \& McKee 1989; (2) detailed balance applied to the reverse reaction; (3) determined by detailed balance from photodetachment cross section of Wishart 1979; (4) Dalgarno \& Lepp 1987; (5) Pineau des Forêts et al. 1989; (6) same as corresponding H reaction; (7) same as corresponding $\mathrm{H}$ reaction but scaled by D reduced mass; (8) Dalgarno \& McDowell 1956, but scaled by D reduced mass; (9) Pineau des Forêts et al. 1986; (10) Zygelman et al. 1989, multiplied by 0.25 to account for approach probability factor (see Stancil \& Zygelman 1996); (11) determined from cross section of Peart \& Hayton 1994; (12) Stancil \& Zygelman 1996, but scaled by D reduced mass; (13) fitted to Kimura, Dutta, \& Shimakura 1994, but scaled by D reduced mass; (14) assumed same as reaction (24); (15) fitted to Ramaker \& Peek 1976; (16) Shapiro \& Kang 1987; (17) fitted to Schneider et al. 1994, 1997; (18) Karpas et al. 1979; (19) Barlow 1994; (20) fitted to Launay, Le Dourneuf, \& Zeippen 1991; (21) fitted to Juřek et al. 1995; (22) Zygelman et al. 1998; (23) fitted to Kraemer, Špirko, \& Juřek 1995 , multiplied by 0.25 to account for approach probability factor (see note 10); (24) determined from cross section of Linder, Janev, \& Botero 1995; (25) Black 1978; (26) Guberman 1994; (27) Datz et al. 1995; (28) fitted to Ramaker \& Peek 1976, but scaled by reduced mass, and Frommhold \& Pickett 1978; (29) determined from cross section of Strömholm et al. 1995; (30) Stancil \& Dalgarno 1997b; (31) Smith et al. 1982; (32) fitted to Zhang \& Miller 1989; (33) Larsson et al. 1996; (34) Millar, Bennett, \& Herbst 1989; (35) assumed same as reaction (80); (36) fitted to Stancil, Babb, \& Dalgarno 1993; (37) estimated from Ivanov \& Skoblo 1988; (38) estimate, Stancil et al. 1993; (39) same as corresponding H reaction estimate of Stancil et al. 1996; (40) fitted to Rawlings, Drew, \& Barlow 1993 and Rawlings 1997, private communication; (41) fitted to Latter \& Black 1991; (42) estimated from Dehmer \& Chupka 1995; and (43) Geddes \& McCullough 1994. 
TABLE 2

Cosmological Model Parameters

\begin{tabular}{llllll}
\hline \hline Parameters & Model I & Model II & Model III & Model IV & Model V \\
\hline$\Omega_{0} \ldots \ldots \ldots \ldots$ & 1.0 & 1.0 & 1.0 & 1.0 & 0.3 \\
$\Omega_{b} \ldots \ldots \ldots$ & 0.1 & 1.0 & 0.0367 & 0.015 & 0.0367 \\
$h \ldots \ldots \ldots \ldots$ & 0.5 & 0.5 & 0.67 & 0.67 & 0.67 \\
$\Omega_{b} h^{2} \ldots \ldots$ & 0.025 & 0.25 & 0.0165 & 0.00673 & 0.0165 \\
$Y \ldots \ldots \ldots \ldots$ & 0.284 & 0.263 & 0.242 & 0.230 & 0.242 \\
$n_{\mathrm{He}} / n_{\mathrm{H}} \ldots \ldots$ & 0.1 & $9.0 \mathrm{E}-2$ & $8.02 \mathrm{E}-2$ & $7.5 \mathrm{E}-2$ & $8.02 \mathrm{E}-2$ \\
$n_{\mathrm{D}} / n_{\mathrm{H}} \ldots \ldots$ & $5.0 \mathrm{E}-5$ & $1.0 \mathrm{E}-4$ & $4.0 \mathrm{E}-5$ & $2.0 \mathrm{E}-4$ & $4.0 \mathrm{E}-5$ \\
$n_{\mathrm{Li}} / n_{\mathrm{H}} \cdots \cdots$ & $1.0 \mathrm{E}-10$ & $1.0 \mathrm{E}-8$ & $2.3 \mathrm{E}-10$ & $1.9 \mathrm{E}-10$ & $2.3 \mathrm{E}-10$ \\
\hline
\end{tabular}

where $\Omega_{b}$ is the ratio of the baryonic-matter density to the critical density required to close the Universe, $h$ is the Hubble constant in units of $100 \mathrm{~km} \mathrm{~s}^{-1} \mathrm{Mpc}^{-1}, Y$ is the helium mass fraction, and the redshift $z$ is related to the time by the expression

$$
\frac{d t}{d z}=\frac{3.086 \times 10^{17}}{h(1+z)^{2}\left(1+\Omega_{0} z\right)^{1 / 2}} \mathrm{~s}^{-1}
$$

(e.g., Peebles 1993). The radiation temperature is given by $T_{r}=2.728(1+z) \mathrm{K}$ (Fixsen et al. 1996). The matter temperature $T_{m}$ is taken from the models of Puy et al. (1993). The $\mathrm{H}(n=2)$ abundances are taken from Rybicki \& Dell'Antonio (1996), who made a careful study of the recombination epoch, including line-trapping of $\mathrm{Ly} \alpha$ photons.

We have investigated five different cosmological models. Their parameters are given in Table 2, and all but Model V incorporate the closure parameter $\Omega_{0}=1.0$. Models I, II, and III are the same as described in Stancil et al. (1996). Model IV is the D-rich model used by Palla et al. (1995) corresponding to the assumed deuterium abundance along the line of sight to the high-redshift $(z=3.32)$ absorption complex toward quasar Q0014+813 (Songaila et al. 1994;
Rugers \& Hogan 1996). Model V is an open universe model with $\Omega_{0}=0.3$ but with all other parameters identical to Model III. While Models I, II, and IV lie outside of the observationally deduced range of the baryonic fraction of the critical density, $0.009 \leq \Omega_{b} h^{2} \leq 0.02$ (Copi, Schramm, $\&$ Turner 1995), we include them for comparison with previous chemical models.

\section{RESULTS AND DISCUSSION}

We determined the fractional abundances of all species listed in Table 3 as a function of redshift for the five different cosmological models. The results for our standard Model III are presented in Figures 1 and 2, while the fractional abundances at $z=10$ for each of the models are given in Table 3 with a comparison to previous calculations. To visualize the primary reaction processes, Figures 3-12 plot the formation and destruction rates (with fractional abundances) for each of the molecules for Model III except $\mathrm{He}_{2}^{+}$. Similar plots have been given by Abel et al. (1997) for $\mathrm{H}^{-}, \mathrm{H}_{2}^{+}$, and $\mathrm{H}_{2}$ and by Bougleux \& Galli (1997) for $\mathrm{LiH}$.

As shown in Figure $3, \mathrm{HeH}^{+}$is primarily formed by the radiative association reactions (T1.37) and (T1.38) with some contribution from (T1.42) peaking near $z \sim 300$. The

TABLE 3

Fractional Abundances $n(x) / n_{\mathrm{H}}$ AT $z=10$ For Models I-V

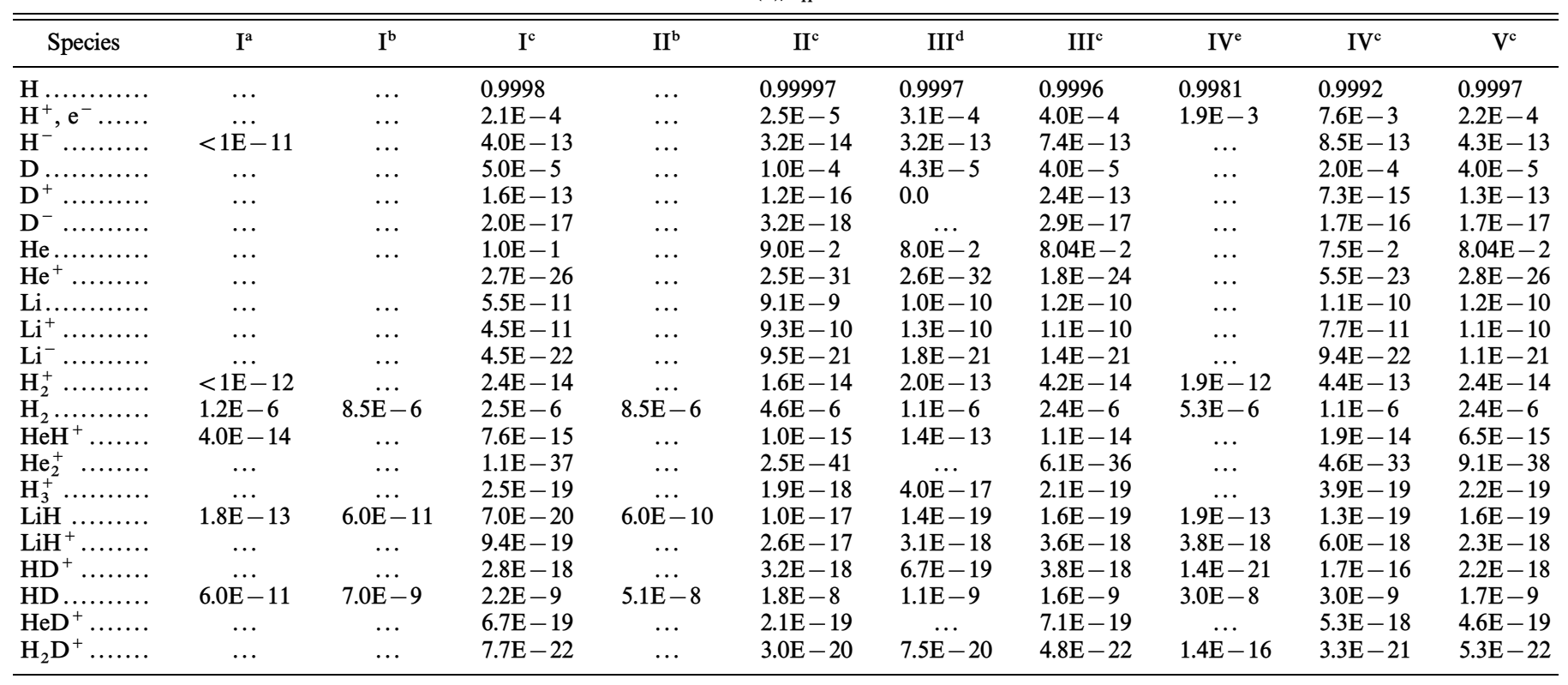

${ }^{a}$ Lepp \& Shull (1984).

b Puy et al. (1993).

c This work.

${ }^{\mathrm{d}} \mathrm{GP}$.

${ }^{\text {e }}$ Palla et al. (1995). 


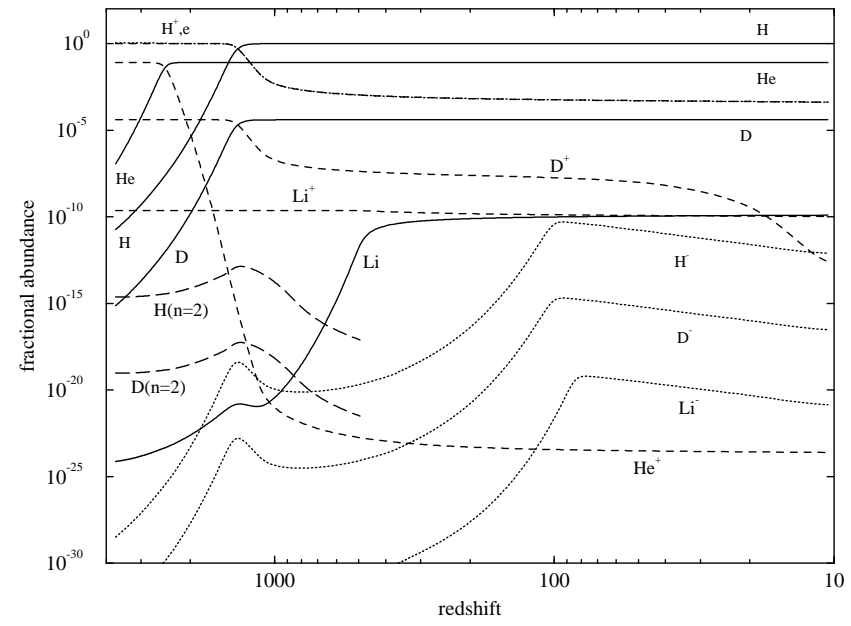

Fig. 1.-Fractional abundances $n(x) / n_{\mathrm{H}}$ of atoms for the Model III chemistry of the recombination era primordial gas. $\Omega_{b} h^{2}=0.0165$, $Y=0.242, n_{\mathrm{He}} / n_{\mathrm{H}}=0.0802, n_{\mathrm{D}} / n_{\mathrm{H}}=4.0 \times 10^{-5}, n_{\mathrm{Li}} / n_{\mathrm{H}}=2.3 \times 10^{-10}(\mathrm{see}$ Table 2 for other parameters).

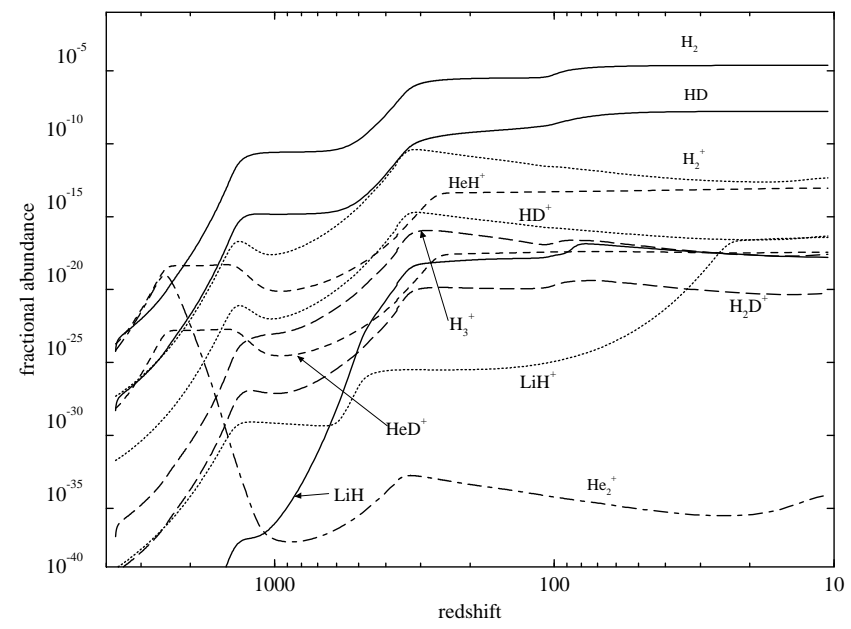

FIG. 2.-Fractional abundances of molecules for the Model III chemistry of the recombination era primordial gas (see Table 2 for parameters).

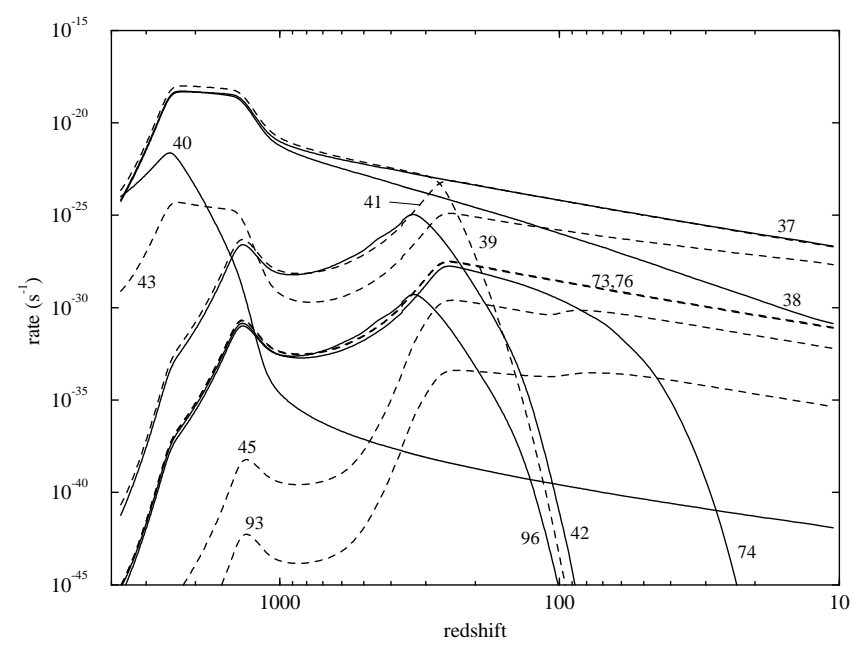

Fig. 3.- Rates of dominant $\mathrm{HeH}^{+}$formation (solid lines) and destruction (dotted lines) processes for Model III. The numbers correspond to the reactions listed in Table 1.

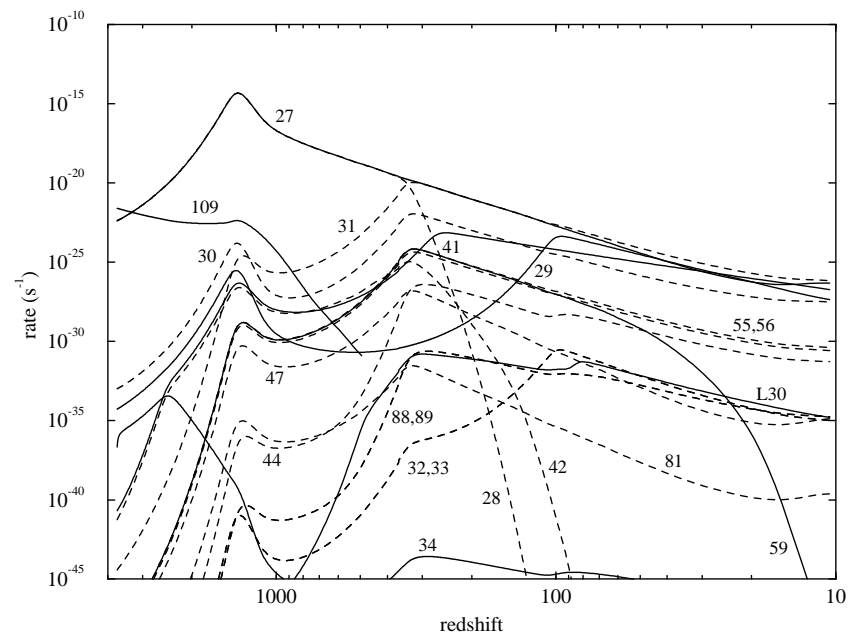

FIG. 4.- Same as Fig. 3 but for $\mathrm{H}_{2}^{+}$

radiative association reaction (T1.40) is only important for $z>3000$ when there is significant $\mathrm{He}^{+}$abundance. $\mathrm{HeH}^{+}$is mainly destroyed by photodissociation (T1.39) for $z \gtrsim 300$ and by collisions with $\mathrm{H}$ (T1.41) and dissociative recombination (T1.43) for $z \lesssim 300$. The remaining reactions play only minor roles in the chemistry. The $\mathrm{HeH}^{+}$chemistry is similar to that used by GP. However, GP adopted the inverse predissociation rate coefficients of Roberge \& Dalgarno (1982) for (T1.37), which are an order of magnitude larger than the results of Jurek, Spirko, \& Kraemer (1995). The Juřek et al. (1995) results have been confirmed by Zygelman et al. (1998). This discrepancy is reflected in the $\mathrm{HeH}^{+}$fractional abundance given in Table 3. With the exception of (T1.37), the major uncertainty in the $\mathrm{HeH}^{+}$ reaction network is (T1.41). The adopted rate coefficient is limited to the ion cyclotron resonance measurement of Karpas, Anicich, \& Huntress (1979). Additional studies of (T1.41), including its vibrational state dependence, are needed.

The formation and destruction processes of $\mathrm{H}_{2}^{+}$are shown in Figure 4. After recombination it is primarily produced by radiative association (T1.27); earlier it is made by associative detachment of $\mathrm{H}$ with $\mathrm{H}(n=2)(\mathrm{T} 1.109)$. For $z \lesssim 100$, (T1.29) and proton abstraction from $\mathrm{HeH}^{+}(\mathrm{T} 1.41)$ contribute significantly and dominate (T1.27) for $z \lesssim 40$. For $z \gtrsim 400$, photodissociation (T1.28) is the primary destruction mechanism, but at later times $\mathrm{H}_{2}^{+}$is mainly removed by charge exchange with $\mathrm{H}$ (T1.31). Dissociative recombination (T1.30) is also a significant destruction path for $z \lesssim 400$. Even though GP adopted a similar chemistry, our $\mathrm{H}_{2}^{+}$abundance is nearly a factor of 5 smaller, as shown in Table 3 at $z=10$. This discrepancy is related to their larger $\mathrm{HeH}^{+}$abundance, since (T1.41) becomes their dominant $\mathrm{H}_{2}^{ \pm}$production mode at late times. In addition to (T1.41), the primary uncertainty in the $\mathrm{H}_{2}^{+}$chemical network is the adopted rate coefficient for (T1.31), which, as is the case for (T1.41), is taken from the measurement of Karpas et al. (1979).

Figure 5 shows that $\mathrm{H}_{2}$ is formed through $\mathrm{H}_{2}^{+}$by (T1.31) for $130 \lesssim z \lesssim 700$ and through $\mathrm{H}^{-}$by (T1.36) for $z \lesssim 130$. Some contributions are made through deuterated species by (T1.64) and (T1.66), and as noted by Latter \& Black (1991), reaction (T1.110) with excited $\mathrm{H}$ forms some $\mathrm{H}_{2}$ for $z \gtrsim$ 1300; however, its abundance is kept low because of the 


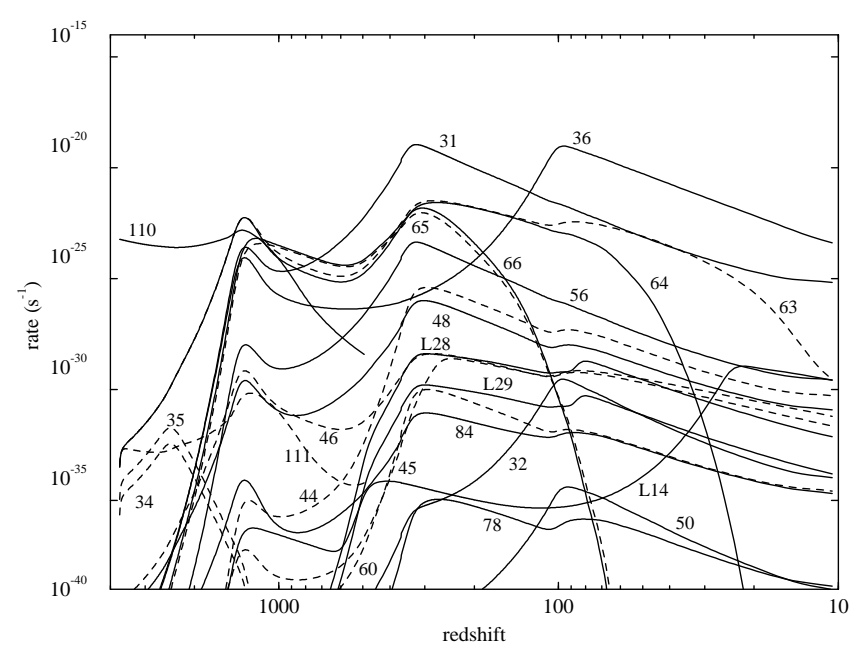

Fig. 5.-Same as Fig. 3 but for $\mathrm{H}_{2}$

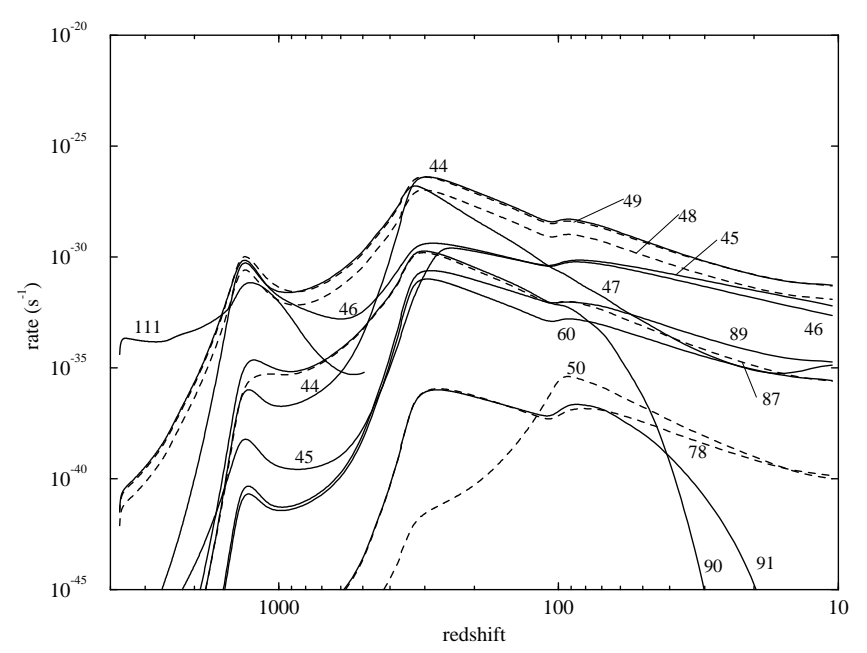

FIG. 6. - Same as Fig. 3 but for $\mathrm{H}_{3}^{+}$

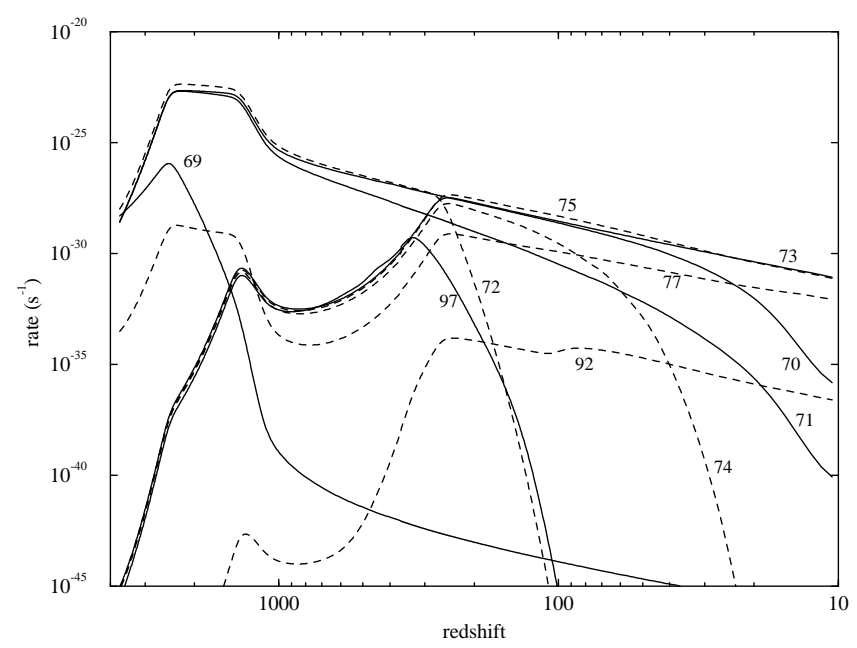

Fig. 7.-Same as Fig. 3 but for $\mathrm{HeD}^{+}$

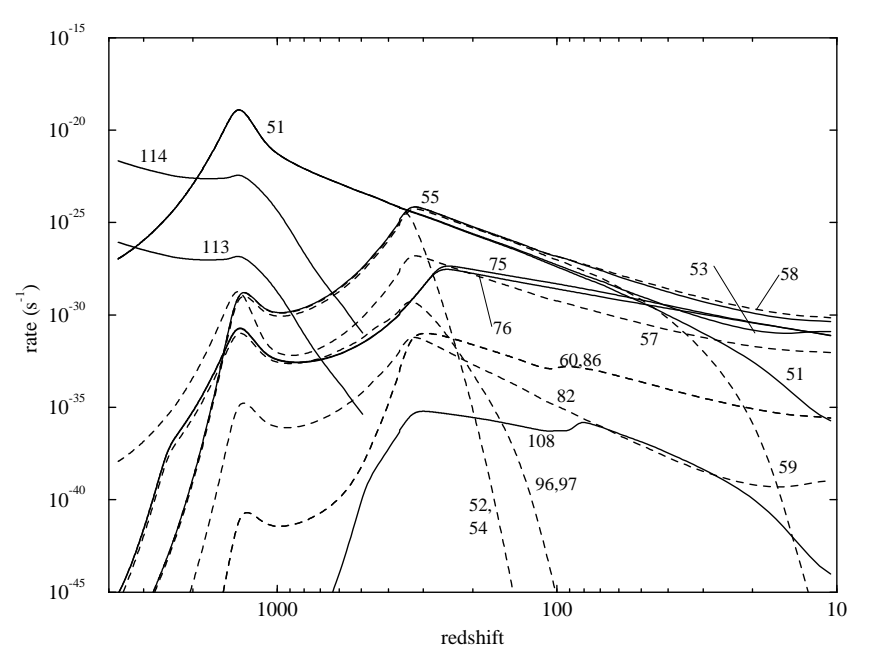

Fig. 8.-Same as Fig. 3 but for $\mathrm{HD}^{+}$

high efficiency of $\mathrm{H}$ photoionization (T1.2). $\mathrm{H}_{2}$ is robust and, because the CBR lacks a consequential UV component, suffers from no significant destruction processes. Only collisions with $\mathrm{D}^{+}$(T1.63) and $\mathrm{D}$ (T1.65) and, for $z \gtrsim 2000$, collisions with $\mathrm{He}^{+}$(T1.34) and (T1.35) are effective. Our computed abundance is in good agreement with GP at $z=10$, since the adopted chemistries are nearly identical. As for $\mathrm{H}_{2}^{+}$, the primary uncertainty is the reliability of the measured rate coefficient for (T1.31).

The most abundant polyatomic molecule formed in the early universe is $\mathrm{H}_{3}^{+}$, and its important reaction rates are displayed in Figure 6. As in interstellar clouds (Martin, McDaniel, \& Meeks 1961; Herbst \& Klemperer 1973), it is primarily produced by (T1.44), but for $z \gtrsim 1500$ the excited $\mathrm{H}$ reaction (T1.111) is the major formation route. (T1.45) and (T1.89) make minor contributions. At all redshifts, $\mathrm{H}_{3}^{+}$ is primarily destroyed by dissociative recombination (T1.49) and (T1.48). Our abundance estimate is more than 2 orders of magnitude smaller than that obtained by GP. The chemistry is comparable, except that GP primarily produce $\mathrm{H}_{3}^{+}$ by the radiative association reaction (T1.46). For (T1.46) they adopted the large rate coefficient $1 \times 10^{-16} \mathrm{~cm}^{3} \mathrm{~s}^{-1}$ recommended by Gerlich \& Horning (1992) based on an ion trap measurement and a classical trajectory analysis. However, the reaction involves rovibrational transitions within the ground electronic state, and experience from fully quantal calculations for diatomic systems such as $\mathrm{HeH}^{+}$, $\mathrm{LiH}^{+}$, and $\mathrm{LiH}$ suggests that the rate coefficient should be much smaller. We prefer the temperature-independent value of $1 \times 10^{-20} \mathrm{~cm}^{3} \mathrm{~s}^{-1}$. Reaction (T1.46) needs to be further investigated before an accurate picture of $\mathrm{H}_{3}^{+}$can be obtained.

As shown in Figure 7, the primary reactions involved in the chemistry of $\mathrm{HeD}^{+}$are similar to those for $\mathrm{HeH}^{+}$. It is produced by radiative association (T1.70) and the exchange reaction (T1.97), and it is destroyed by photodissociation (T1.72) and the exchange reaction (T1.75). Additionally, the $\mathrm{D}$ exchange reaction (T1.73) is an important $\mathrm{HeD}^{+}$production mechanism. The abundance of $\mathrm{HeD}^{+}$tracks that of $\mathrm{HeH}^{+}$. The chemistry is rather uncertain, because all of the adopted rate coefficients are taken from corresponding $\mathrm{He}-\mathrm{H}$ reactions but scaled by the collision system reduced mass. Similar to $\mathrm{HeH}^{+}$, the most uncertain reaction is that of (T1.75). No previous abundance estimates of $\mathrm{HeD}^{+}$have been reported. We did not consider ${ }^{3} \mathrm{He}$ since its chemistry 


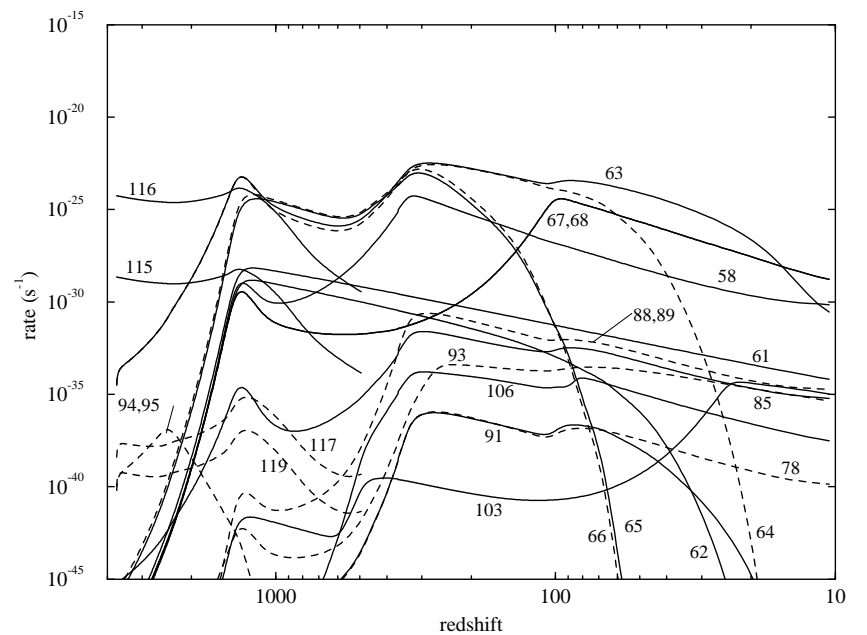

FIG. 9.-Same as Fig. 3 but for HD

is not expected to be different from that of ${ }^{4} \mathrm{He}$. The $\mathrm{He}$ reaction analogous to (T1.8) (Dutta, Lane, \& Kimura 1992) is unimportant, since the $\mathrm{He}^{+}$abundance is negligible for $z<1000$.

Figure 8 shows that the reaction network for $\mathrm{HD}^{+}$is comparable to that of $\mathrm{H}_{2}^{+}$. It is produced by the radiative association processes (T1.51) and (T1.53), $\mathrm{D}^{+}$abstraction from $\mathrm{HeD}^{+}$(T1.75), and proton abstraction from $\mathrm{HeH}^{+}$ (T1.76). $\mathrm{HD}^{+}$is removed by photodissociation (T1.52) and (T1.54) for $z \gtrsim 400$ and by charge exchange (T1.58) and dissociative recombination (T1.57) for $z \lesssim 400$. However, the exchange reaction (T1.55), for which there is no comparable process in the $\mathrm{H}_{2}^{+}$formation scheme, becomes the dominant formation mechanism for $z \lesssim 400$. Our computed $\mathrm{HD}^{+}$abundance is larger than that of GP, since they neglected (T1.55), (T1.75), and (T1.76). Most of the chemistry is uncertain, because only dissociative recombination (T1.57) has been explicitly investigated, while the remaining reaction rates were deduced from the corresponding hydrogen chemistry. Further studies of (T1.55) and (T1.58) are of most importance.

Similar to $\mathrm{H}_{2}, \mathrm{HD}$ is produced by associative detachment (T1.67) and (T1.68) for $z \lesssim 130$, charge exchange (T1.58) for $130 \lesssim z \lesssim 600$, and excited $H$ reactions (T1.115) and (T1.116) for $z \gtrsim 1000$, as shown in Figure 9. However, the

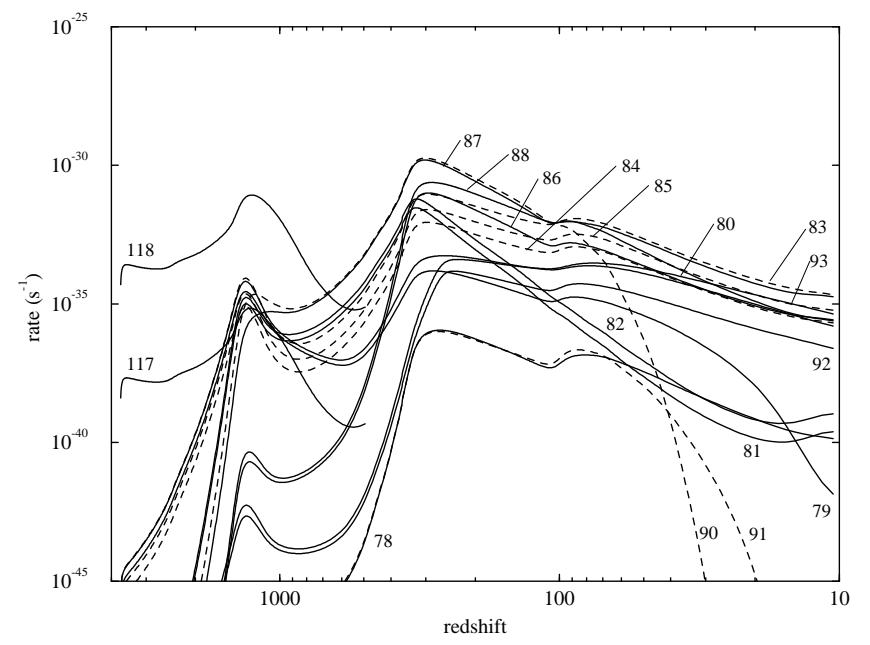

FIG. 10.- Same as Fig. 3 but for $\mathrm{H}_{2} \mathrm{D}^{+}$

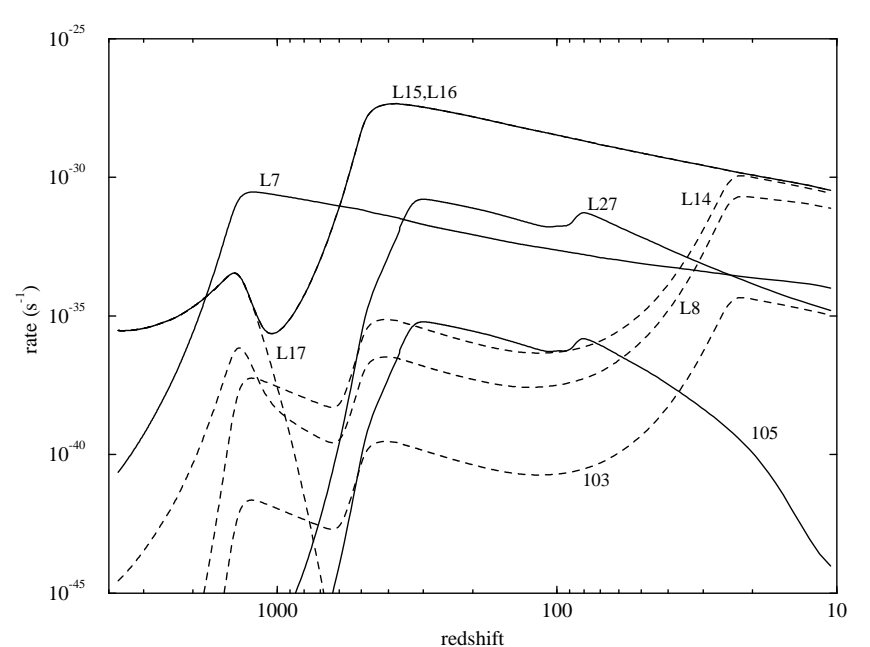

Fig. 11.-Same as Fig. 3 but for $\mathrm{LiH}^{+}$

primary HD formation process is actually the D exchange reactions (T1.63) and (T1.65) with the reverse D exchange reactions (T1.64) and (T1.66) being the dominant destruction routes. Quite good agreement is found between the current abundance estimates and that obtained by GP. The adopted rate coefficients of (T1.63) and (T1.64) are from the flow-tube measurements of Smith, Adams, \& Alge (1982), while those of (T1.65) and (T1.66) are based on the calculations of Zhang \& Miller (1989). Further studies are needed to reduce the uncertainty in the HD abundance.

$\mathrm{H}_{2} \mathrm{D}^{+}$is formed by (T1.88) and (T1.86) for $z \lesssim 1000$, (T1.93) for $z \lesssim 20$, and (T1.117) and (T1.118) for $z \gtrsim 1000$. It is destroyed by dissociative recombination (T1.83)-(T1.85). However, unlike $\mathrm{H}_{3}^{+}$, (T1.87) and (T1.90) are the primary formation and destruction mechanisms for $130 \lesssim z \lesssim 1000$, as displayed in Figure 10. The discrepancy with the abundance estimates of GP is a consequence of their enhanced $\mathrm{H}_{3}^{+}$abundance. The other major uncertainties in the $\mathrm{H}_{2} \mathrm{D}^{+}$ chemistry are due to the limited information on (T1.87), (T1.88), and (T1.90).

For completeness, we also show in Figures 11 and 12 the primary formation and destruction processes for $\mathrm{LiH}^{+}$and $\mathrm{LiH}$, respectively. $\mathrm{LiH}^{+}$is primarily formed by the radiative

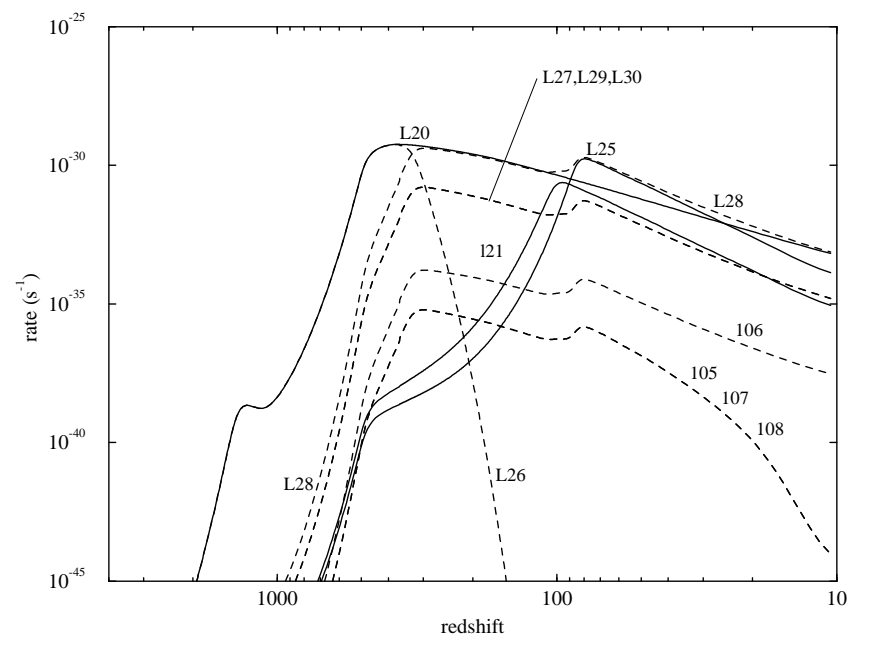

Fig. 12.-Same as Fig. 3 but for $\mathrm{LiH}$ 
TABLE 4

Deuterium Fractionation at $z=10$ for Models I-V

\begin{tabular}{|c|c|c|c|c|c|c|c|c|c|c|}
\hline Species & $\mathrm{I}^{\mathrm{a}}$ & $\mathrm{I}^{\mathrm{b}}$ & $I^{c}$ & II $^{\mathrm{b}}$ & $\mathrm{II}^{\mathrm{c}}$ & III $^{d}$ & III $^{\mathrm{c}}$ & $\mathrm{IV}^{\mathrm{e}}$ & $\mathrm{IV}^{\mathrm{c}}$ & $\mathrm{V}^{\mathrm{c}}$ \\
\hline $\mathrm{D}^{+} / \mathrm{H}^{+}$ & $\ldots$ & $\ldots$ & $7.5 \mathrm{E}-10$ & $\ldots$ & $4.8 \mathrm{E}-12$ & 0 & $6.0 \mathrm{E}-10$ & $\ldots$ & $9.6 \mathrm{E}-12$ & $6.0 \mathrm{E}-10$ \\
\hline $\mathrm{D}^{-} / \mathrm{H}^{-}$ & $\ldots$ & $\ldots$ & $5.0 \mathrm{E}-5$ & $\ldots$ & $1.0 \mathrm{E}-4$ & $\ldots$ & $4.0 \mathrm{E}-5$ & $\ldots$ & $2.0 \mathrm{E}-4$ & $4.0 \mathrm{E}-5$ \\
\hline $\mathrm{HD} / \mathrm{H}_{2} \ldots \ldots$. & $5.0 \mathrm{E}-5$ & $8.2 \mathrm{E}-4$ & $8.9 \mathrm{E}-4$ & $6.0 \mathrm{E}-3$ & $3.8 \mathrm{E}-3$ & $1.0 \mathrm{E}-3$ & $6.9 \mathrm{E}-4$ & $5.7 \mathrm{E}-3$ & $2.8 \mathrm{E}-3$ & $7.0 \mathrm{E}-4$ \\
\hline $\mathrm{HeD}^{+} / \mathrm{HeH}^{+}$. & $\ldots$ & $\ldots$ & $8.9 E-5$ & $\ldots$ & $2.0 \mathrm{E}-4$ & & $6.6 \mathrm{E}-5$ & & $2.8 \mathrm{E}-4$ & $7.1 \mathrm{E}-5$ \\
\hline $\mathrm{H}_{2} \mathrm{D}^{+} / \mathrm{H}_{3}^{+} \ldots \ldots$ & $\ldots$ & $\ldots$ & $3.1 \mathrm{E}-3$ & $\ldots$ & $1.6 \mathrm{E}-2$ & $1.9 \mathrm{E}-3$ & $2.2 \mathrm{E}-3$ & $5.2 \mathrm{E}-3$ & $8.6 \mathrm{E}-3$ & $2.4 \mathrm{E}-3$ \\
\hline
\end{tabular}

${ }^{a}$ Lepp \& Shull (1984).

${ }^{b}$ Puy et al. (1993).

${ }^{c}$ This work.

${ }^{\mathrm{d}}$ GP.

${ }^{\mathrm{e}}$ Palla et al. (1995).

association reactions (L7) ${ }^{7}$ and (L15) and destroyed by photodissociation (L16) and (L17). However, for $z \lesssim 25$, the exchange reaction (L14) and dissociative recombination (L8) are the important destruction mechanisms. Radiative association (L20) and associative detachment (L25) are the main production mechanisms for $\mathrm{LiH}$, while photodissociation (L26) and collisions with H (L28) are the dominant removal routes. The current abundances of $\mathrm{LiH}^{+}$and $\mathrm{LiH}$ are in good agreement with GP. GP also include $\mathrm{LiH}$ formation from excited $\mathrm{Li}$ radiative association, but Bougleux \& Galli (1997) have shown that it only contributes for $z \gtrsim 650$. Reactions (L28), (L25), (L14), and (L8) are the major uncertainties.

We have run other cosmological models for comparison with previous calculations. The current $\mathrm{H}_{2}$ abundance is in good agreement with the results of Lepp \& Shull (1984) for Model I but is nearly a factor of 4 smaller than Puy et al. (1993). The Lepp \& Shull HD abundance appears to be too small, while that of Puy et al. is too large. Both previous calculations found erroneously large $\mathrm{LiH}$ abundances because of the adoption of a large radiative association rate coefficient.

For the baryonic $\left(\Omega_{b}=1\right)$ universe model II, our $\mathrm{H}_{2}, \mathrm{HD}$, and $\mathrm{LiH}$ abundances are smaller than those obtained by Puy et al. (1993), but larger than any of our other models mostly as a consequence of a higher density and enhanced $\mathrm{D}$ and $\mathrm{Li}$ primordial abundances. The cause of the large LiH discrepancy is the same as discussed for Model I. As noted by Palla et al. (1995), the enhanced D model (Model IV) has little effect on $\mathrm{H}_{2}$ but increases the deuterium species abundances: $\mathrm{HD}^{+}, \mathrm{HeD}^{+}, \mathrm{HD}$, and $\mathrm{H}_{2} \mathrm{D}^{+}$. The open universe Model $\mathrm{V}$ results in a slightly reduced ionization fraction due to the increase in time per unit redshift (see eq. [23]) as compared to Model III. The abundances of all the ions are decreased except for $\mathrm{Li}^{+}, \mathrm{H}_{3}^{+}$, and $\mathrm{H}_{2} \mathrm{D}^{+}$.

Table 4 presents the deuterium fractionation. For Model III we find the isotope abundance ratios $n\left(\mathrm{HD}^{+}\right) / n\left(\mathrm{H}_{2}^{+}\right)$, $n(\mathrm{HD}) / n\left(\mathrm{H}_{2}\right)$, and $n\left(\mathrm{H}_{2} \mathrm{D}^{+}\right) / n\left(\mathrm{H}_{3}^{+}\right)$to be enhanced compared to the primordial abundance ratio $n_{\mathrm{D}} / n_{\mathrm{H}}=4 \times 10^{-5}$ by factors of up to $\sim 50$. Conversely, the $n\left(\mathrm{D}^{+}\right) / n\left(\mathrm{H}^{+}\right)$ratio is reduced by a factor of about $10^{5}$, since for $z<40, \mathrm{D}^{+}$is significantly depleted because of the charge transfer reaction (T1.9). The reverse reaction (T1.8) is endothermic by 41 $\mathrm{K}$. Comparing to GP, the ratios $n(\mathrm{HD}) / n\left(\mathrm{H}_{2}\right)$ and

\footnotetext{
${ }^{7}$ The reaction labels $(L x)$ in the figures correspond to process $(x)$ in Stancil et al. (1996), where the lithium chemistry was fully discussed.
}

$n\left(\mathrm{H}_{2} \mathrm{D}^{+}\right) / n\left(\mathrm{H}_{3}^{+}\right)$are in fair agreement, but the $\mathrm{HD}^{+}$discrepancy persists for $n\left(\mathrm{HD}^{+}\right) / n\left(\mathrm{H}_{2}^{+}\right)$.

The enhanced $\mathrm{HD} / \mathrm{H}_{2}$ fractionation can be attributed to reactions (T1.63) and (T1.65). The endothermicity of reaction (T1.90) with respect to (T1.87) and the smaller rate coefficients of (T1.83)-(T1.85) compared to dissociative recombination of $\mathrm{H}_{3}^{+}$account for the $\mathrm{H}_{2} \mathrm{D}^{+} / \mathrm{H}_{3}^{+}$fractionation. While reactions (T1.78) and (T1.91) are important deuterium fractionation mechanisms in interstellar clouds (Dalgarno \& Lepp 1984), they are negligible here; the reactions may play a role in collapsing primordial clouds.

\section{IMPLICATIONS FOR CBR ANISOTROPIES}

Dubrovich (1993) has suggested that Thomson scattering of CBR photons with $\mathrm{H}_{2} \mathrm{D}^{+}$in early epochs might provide for suppression of spatial anisotropies in the CBR spectrum. He inferred that the $\mathrm{H}_{2} \mathrm{D}^{+}$opacity may be near unity since it can be formed through reaction (T1.78), leading to significant fractionation $n\left(\mathrm{H}_{2} \mathrm{D}^{+}\right) / n\left(\mathrm{H}_{3}^{+}\right)$much greater than the primordial deuterium abundance. We find that while the fractionation is about 50, the abundances of both $\mathrm{H}_{3}^{+}$and $\mathrm{H}_{2} \mathrm{D}^{+}$are small. It was previously pointed out by Palla et al. (1995) that the $\mathrm{H}_{2} \mathrm{D}^{+}$optical depth is $<10^{-9}$ and therefore would have a negligible effect on the CBR spectrum. Our $\mathrm{H}_{2} \mathrm{D}^{+}$abundance is much smaller than that obtained by Palla et al., suggesting an optical depth of $\lesssim 10^{-14}$ but confirming their conclusion. We also note that (T1.78) plays an insignificant role in $\mathrm{H}_{2} \mathrm{D}^{+}$production in the early universe, as shown in Figure 10.

Dubrovich \& Lipovka (1995) have investigated the effect of $\mathrm{H}_{2} \mathrm{D}^{+}$on distortions of the CBR spectrum for $z=10-30$. The detection of these features could be used to determine the epochs of reionization and reheating $(z \sim 7$ and 10 , respectively; e.g., Gnedin \& Ostriker 1997) and could possibly place constraints on the primordial deuterium abundance. Assuming an $\mathrm{H}_{2} \mathrm{D}^{+}$fractional abundance of $10^{-8}$, Dubrovich \& Lipovka (1995) obtained intensity contrasts in the Rayleigh-Jeans region of the CBR of $<10^{-7}$. Since the intensity contrast scales linearly with abundance, the present calculations suggest $\mathrm{H}_{2} \mathrm{D}^{+}$had an insignificant effect on the CBR distortions, precluding the possibility of obtaining any interesting cosmological information.

\section{CONCLUSIONS}

In conjunction with our previous investigation of the lithium chemistry, a comprehensive survey of all the relevant reactions in the recombination era of the early universe 
for the primordial elements has been performed. The computed abundances of 23 atomic and molecular species for a standard Big Bang cosmogony are generally in good agreement with the recent calculation of GP. However, because of some differences in the adopted rate coefficients, discrepancies remain for the less abundant molecular ions $\mathrm{H}_{2}^{+}$, $\mathrm{HeH}^{+}, \mathrm{H}_{3}^{+}, \mathrm{HD}^{+}$, and $\mathrm{H}_{2} \mathrm{D}^{+}$. To resolve these discrepancies, additional studies of reactions (T1.41), (T1.46), (T1.55), (T1.58), (T1.64), (T1.66), (T1.87), (T1.88), and (T1.90) are necessary.

For $\mathrm{H}_{2}$, uncertainty remains in its formation because of limited information on the charge exchange reaction (T1.31) at low energies. Additional investigations of the $\mathrm{D}$ exchange reactions (T1.63) and (T1.65), the primary formation mechanisms of $\mathrm{HD}$, are needed. Because $\mathrm{H}_{2}$ and $\mathrm{HD}$ are predicted to be the most abundant molecules, an accurate determination of their abundances in the postrecombination era is vital. They are expected to be the dominant coolants in the collapse of the first bound objects (Palla, Salpeter, \& Stahler 1983; Lepp \& Shull 1984).

This work was supported by the U.S. Department of Energy under Contract DE-AC05-96OR22464 to Lockheed Martin Energy Research Corp. (P. C. S.), the National Science Foundation, Cooperative Agreement OSR 9353227 (S. L.), and Division of Astronomical Sciences Grant AST 93-01099 (A. D.). We thank T. Abel for helpful discussions, D. Galli and F. Palla for providing their paper and data prior to publication, and the referee, J. M. Shull, for helpful suggestions.
Abel, T., Anninos, P., Zhang, Y., \& Norman, M. L. 1997, NewA, 2, 181

Barlow, S. G. 1984, Ph.D. thesis, Univ. Colorado

Black, J. H. 1978, ApJ, 222, 125

Bougleux, E., \& Galli, D. 1997, MNRAS, 288, 638

Burles, S., \& Tytler, D. 1996, AJ, 114, 1330

Carswell, R. F., Rauch, M., Weymann, R. J., Cooke, A. J., \& Webb, J. K. 1994, MNRAS, 268, L1

Carswell, R. F., et al. 1996, MNRAS, 278, 518

Copi, C. J., Schramm, D. N., \& Turner, M. S. 1995, Science, 267, 192

Dalgarno, A., \& Fox, J. L. 1994, in Unimolecular and Bimolecular Reaction Dynamics, ed. C. Y. Ng, T. Baer, \& I. Powis (Chichester: Wiley), 1

Dalgarno, A., \& Lepp, S. 1984, ApJ, 287, L47

. 1987, in Astrochemistry, ed. S. P. Tarafdar \& M. P. Varshni (Dordrecht: Reidel), 109

Dalgarno, A., \& McDowell, M. R. C. 1956, Proc. Phys. Soc. London A, 69, 615

Dalgarno, A., Weisheit, J. C., \& Black, J. H. 1973, Astrophys. Lett., 14, 77

Datz, S., et al. 1995, Phys. Rev. Lett., 74, 876

Dehmer, P. M., \& Chupka, W. A. 1995, J. Phys. Chem., 99, 1686

Dubrovich, V. K. 1993, Astron. Lett., 19, 53

Dubrovich, V. K., \& Lipovka, A. A. 1995, A\&A, 296, 301

Dutta, C. M., Lane, N. F., \& Kimura, M. 1992, Phys. Rev. A, 46, 3889

Fixsen, D. J., Cheng, E. S., Gales, J. M., Mather, J. C., Shafer, R. A., \& Wright, E. L. 1996, ApJ, 473, 576

Frommhold, L., \& Pickett, H. M. 1978, Chem. Phys., 28, 441

Galli, D., \& Palla, F. 1998, A\&A, 335, 403

Gaur, V. P., \& Tripathi, B. M. 1985, J. Quant. Spectrosc. Radiat. Transfer, 33,291

Geddes, J. \& McCullough, R. W. 1993, J. Phys. B, 26, L165

Gerlich, D., \& Horning, S. 1992, Chem. Rev., 92, 1509

Gnedin, N. Y., \& Ostriker, J. 1997, ApJ, 486, 581

Guberman, S. L. 1994, Phys. Rev. A, 49, R4277

Herbst, E., \& Klemperer, W. 1973, ApJ, 185, 505

Hollenbach, D., \& McKee, C. F. 1989, ApJ, 342, 306

Irwin, A. W. 1981, ApJS, 45, 624

Ivanov, V. A. \& Skoblo, Yu. E. 1988, Opt. Spectrosc., 65, 445

Juřek, M., Spirko, V., \& Kraemer, W. P. 1995, Chem. Phys., 193, 287

Karpas, Z., Anicich, V., \& Huntress, W. T. 1979, J. Chem. Phys., 70, 2877

Kimura, M., Dutta, C. M., \& Shimakura, N. 1994, ApJ, 430, 435

Kraemer, W. P., Spirko, V., \& Juřek, M. 1995, Chem. Phys. Lett., 236, 177

Larsson, M., et al. 1996, A\&A, 309, L1

Latter, W. B. 1989, Ph. D. thesis, Univ. Arizona

Latter, W. B., \& Black, J. H. 1991, ApJ, 372, 161

Launay, J. M., Le Dourneuf, M., \& Zeippen, C. J. 1991, A\&A, 252, 842

\section{REFERENCES}

Lepp, S., \& Shull, J. M. 1984, ApJ, 280, 465

Linder, F., Janev, R. K., \& Botero, J. 1995, in Atomic and Molecular Processes in Fusion Edge Plasmas, ed. R. K. Janev (New York: Plenum Press), 397

Martin, D. W., McDaniel, E. W., \& Meeks, M. L. 1961, ApJ, 134, 1012

Millar, T. J., Bennett, A., \& Herbst, E. 1989, ApJ, 340, 906

Palla, F., Galli, D., \& Silk, J. 1995, ApJ, 451, 44

Palla, F., Salpeter, E. E., \& Stahler, S. W. 1983, ApJ, 271, 632

Peart, B., \& Hayton, D. A. 1994, J. Phys. B, 27, 2551

Peebles, P. J. E. 1993, Principles of Physical Cosmology (Princeton: Princeton Univ. Press)

Pineau des Forêts, G., Flower, D. R., Hartquist, T. W., \& Dalgarno, A. 1986, MNRAS, 220, 801

Pineau des Forêts, G., Roueff, E., \& Flower, D. R. 1989, MNRAS, 240, 167

Puy, D., Alecian, G., Le Bourlot, J., Léorat, J., \& Pineau des Forêts, G. 1993, A\&A, 267, 337

Ramaker, D. E., \& Peek, J. M. 1976, Phys. Rev. A, 13, 58

Rawlings, J. M. C., Drew, J. E. \& Barlow, M. J. 1993, MNRAS, 265, 968

Roberge, W., \& Dalgarno, A. 1982, ApJ, 255, 489

Rodgers, S. D., \& Millar, T. J. 1996, MNRAS, 280, 1046

Rugers, M., \& Hogan, C. J. 1996, ApJ, 459, L1

Rybicki, G. B., \& Dell'Antonio, I. P. 1996, BAAS, 188, 3202

Sauval, A. J., \& Tatum, J. B. 1984, ApJS, 56, 193

Schneider, I. F., Dulieu, O., Giusti-Suzor, A., \& Roueff, E. 1994, ApJ, 424 983

1997, ApJ, 486, 580

Shapiro, P. R., \& Kang, H. 1987, ApJ, 318, 32

Smith, D., Adams, N. G., \& Alge, E. 1982, ApJ, 263, 123

Songaila, A., Cowie, L. L., Hogan, C. J., \& Rugers, M. 1994, Nature, 368, 599

Stancil, P. C., Babb, J. F., \& Dalgarno, A. 1993, ApJ, 414, 672

Stancil, P. C., \& Dalgarno, A. 1997a, ApJ, 479, 543 1997b, ApJ, 490, 76

1998, Faraday Disc., 109, 61

Stancil, P. C., Lepp, S., \& Dalgarno, A. 1996, ApJ, 458, 401

Stancil, P. C., \& Zygelman, B. 1996, ApJ, 472, 102

Strömholm, C., et al. 1995, Phys. Rev. A, 52, R4320

Tytler, D., Fan, X.-M., \& Burles, S. 1996, Nature, 381, 207

Wishart, A. W. 1979, MNRAS, 187, 59

Zhang, J. Z. H., \& Miller, W. H. 1989, J. Chem. Phys., 91, 1528

Zygelman, B., Dalgarno, A., Kimura, M., \& Lane, N. F. 1989, Phys. Rev. A, 40,2340

Zygelman, B., Stancil, P. C., \& Dalgarno, A. 1998, ApJ, 508, 151 